Universidad deValladolid

PROGRAMA DE DOCTORADO:

INVESTIGACIÓN EN CIENCIAS DE LA SALUD

TESIS DOCTORAL:

\title{
ANÁLISIS DE LAS COMPLICACIONES Y DEL COSTE DE LA INCORRECTA IMPLEMENTACIÓN DEL PROTOCOLO DE PROFILAXIS ANTITROMBÓTICA EN PROCEDIMIENTOS INVASIVOS PROGRAMADOS EN PACIENTES EN TRATAMIENTO ANTICOAGULANTE ORAL CON ACENOCUMAROL
}

Presentada por $\mathbf{M}^{\mathrm{a}}$ Victoria Cuevas Ruiz para optar al grado de

Doctor/a por la Universidad de Valladolid

Dirigida por:

Dra. Jorge Labrador Gómez 
Dedicatoria

A mi familia 


\section{Agradecimientos}

Al doctor Jorge Labrador, director de esta tesis doctoral por su implicación y apoyo incondicional.

A la doctora Beatriz Cuevas, por su inestimable ayuda en la revisión y corrección del manuscrito.

A la doctora Ma Jesús Coma, responsable de la Unidad de Investigación por el apoyo logístico recibido y sus ánimos para iniciar este proyecto.

A la Fundación Burgos por la Investigación de la Salud, cuya ayuda en forma de beca a este proyecto facilitó la realización del mismo.

Al Servicio de Hematología-Hemoterapia del Hospital Universitario de Burgos, cuyos miembros me han alentado a continuar con este proyecto.

A todos los pacientes en tratamiento anticoagulante oral que me han permitido profundizar en el estudio de este tratamiento. 


\section{ABREVIATURAS}

ACV: accidente cerebro vascular.

AVK: antagonista de la vitamina $\mathrm{K}$

FA: fibrilación auricular.

F II: protrombina.

F V: factor V.

F Va: factor $V$ activado.

F VII: factor VII.

F VIII: factor VIII.

F VIIla: factor VIII activado.

F IX: factor IX.

F IXa: factor IX activado.

F X: factor $X$.

F Xa: factor $\mathrm{X}$ activado.

HBPM: heparina de bajo peso molecular.

HNF: heparina no fraccionada.

HS: heparina estándar

HUBU: Hospital Universitario de Burgos.

ISI: Índice de Sensibilidad Internacional

INR: Razón Normalizada Internacional.

OMS: Organización Mundial de la Salud.

TAO: tratamiento anticoagulante oral.

TEP: tromboembolismo pulmonar.

TP: tiempo de protrombina.

TRT: tiempo en rango terapéutico.

TVP: trombosis venosa profunda. 


\section{ÍNDICE}

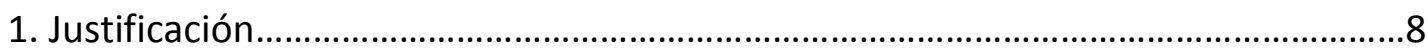

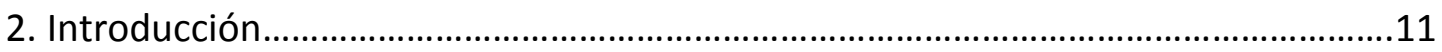

2.1. Introducción al descubrimiento de los anticoagulantes orales

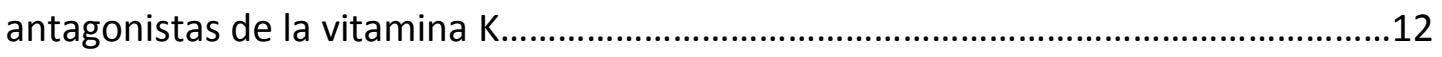

2.1.1. Uso de los anticoagulantes orales antivitamina K en humanos..........................15

2.2. Clasificación de los anticoagulante orales antivitamina K......................................16

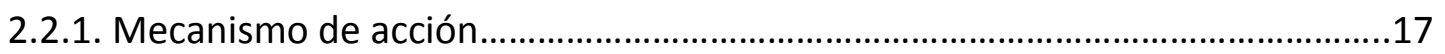

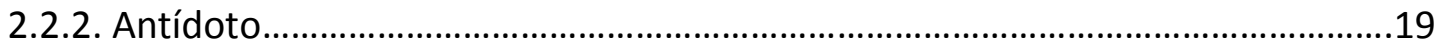

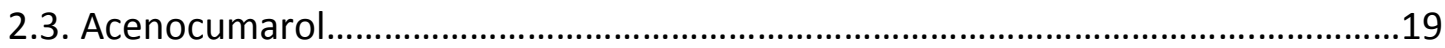

2.4. Control analítico de los anticoagulantes orales antivitamina K..............................20

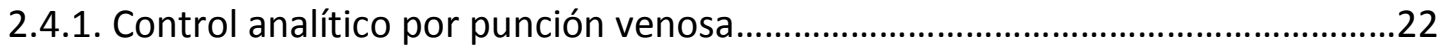

2.4.2. Control analítico por punción digital....................................................................22

2.4.2.1 Coagulómetros portátiles: CoaguChek ${ }^{\circledR}$ XS....................................................23

2.4.2.2. Coste del control con coagulómetros portátiles...............................................24

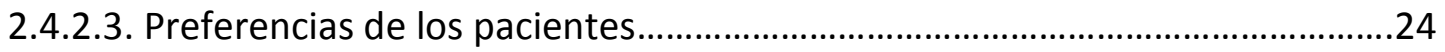

2.4.2.4. Ventajas del uso del coagulómetro portátil...................................................24

2.5. Descentralización del tratamiento anticoagulante oral..........................................25

2.5.1. Programas para la gestión del tratamiento anticoagulante oral.........................25

2.6. Control terapéutico de los anticoagulantes orales

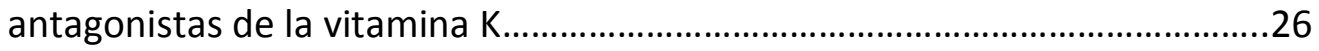

2.6.1. Unidad de control del tratamiento anticoagulante oral...................................26

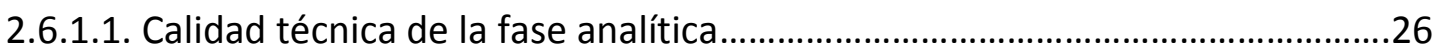

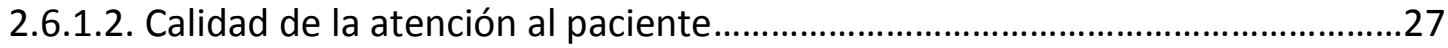

2.7. Control de los anticoagulante orales antagonistas de la vitamina $\mathrm{K}$.....................28

2.7.1. Control terapéutico de los anticoagulante orales

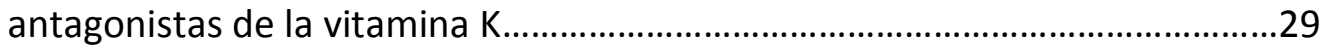

2.8. Indicaciones del tratamiento anticoagulante oral..................................................

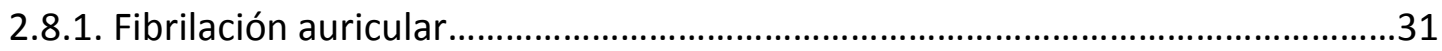

2.8.1.1. Epidemiología......................................................................................................

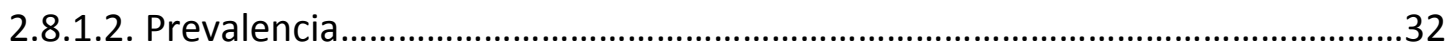




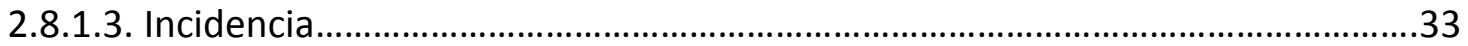

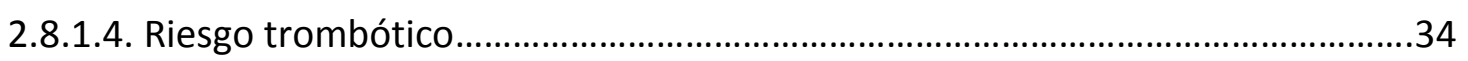

2.8.1.5. Tratamiento anticoagulante oral en Fibrilación Auricular..................................34

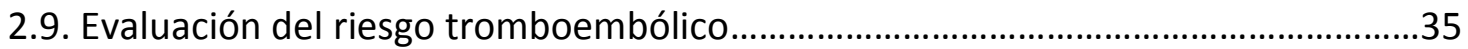

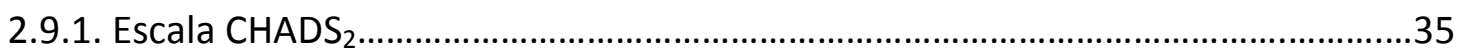

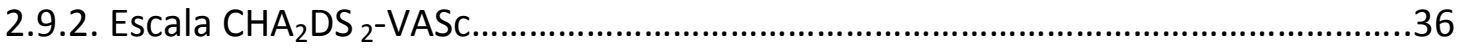

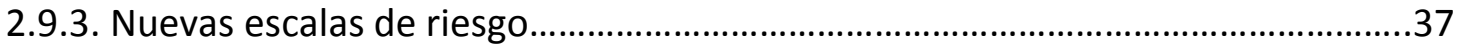

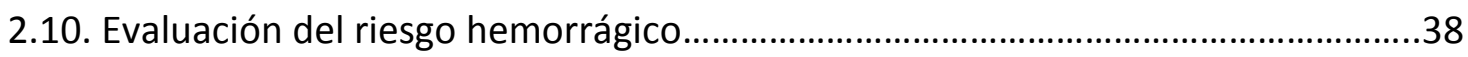

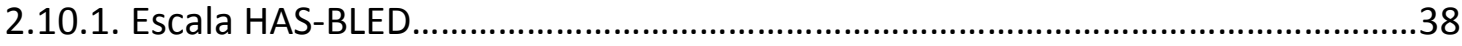

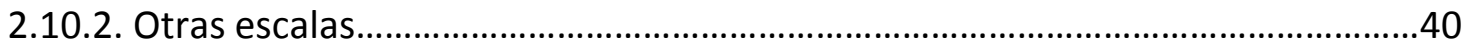

2.11. Manejo del tratamiento anticoagulante oral en el periprocedimiento..................40

2.11.1. Evaluación del riesgo periprocedimiento...............................................................40

2.11.2. Manejo del tratamiento anticoagulante oral en el perioperatorio......................43

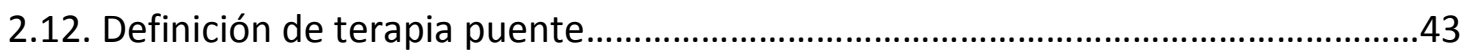

2.13. Heparina de bajo peso molecular.............................................................................44

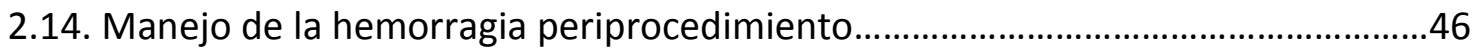

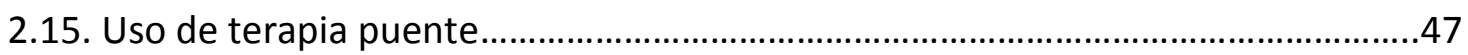

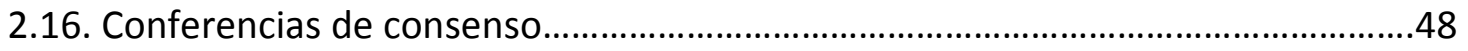

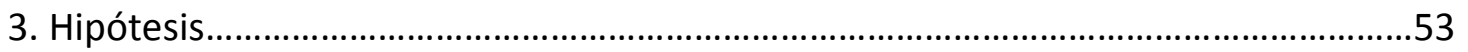

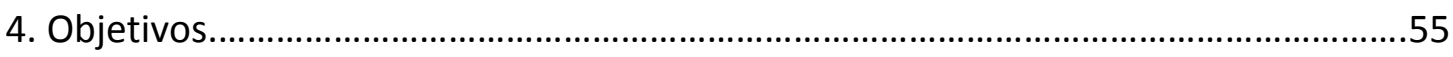

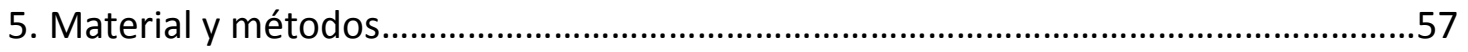

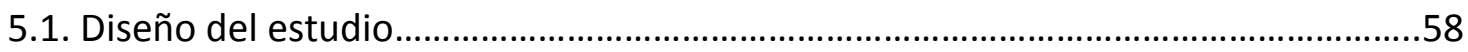

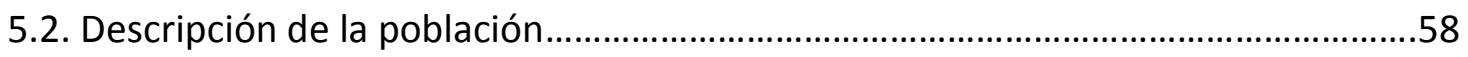

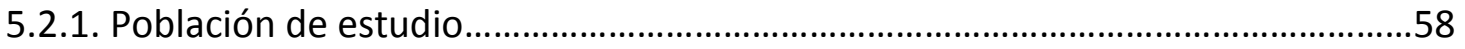

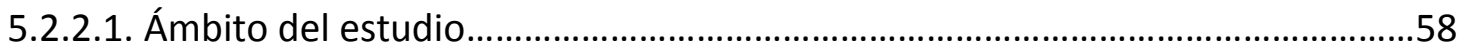

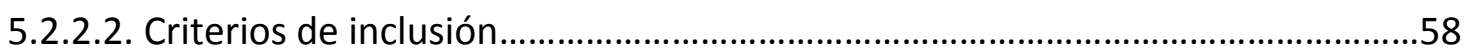

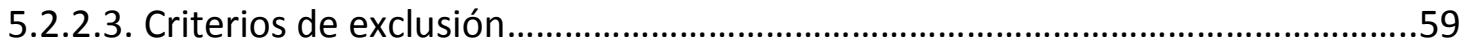

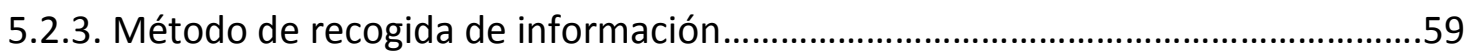

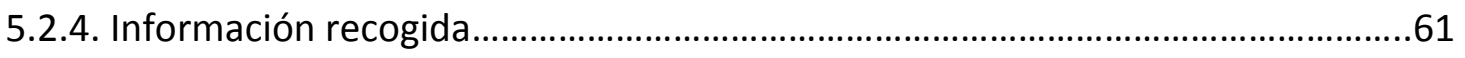

5.3. Método de recogida y tratamiento de los datos.....................................................61

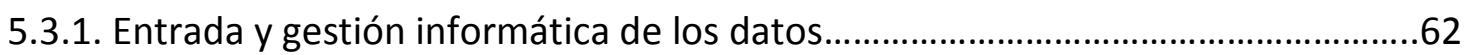

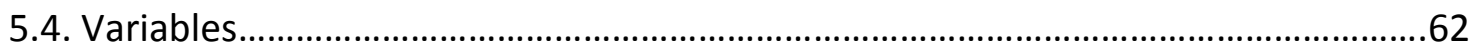




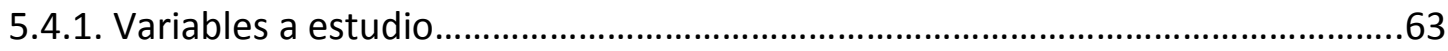

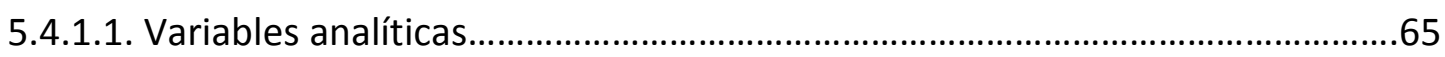

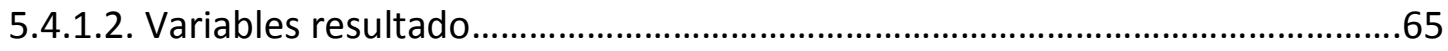

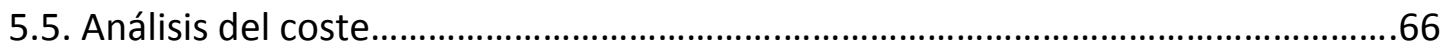

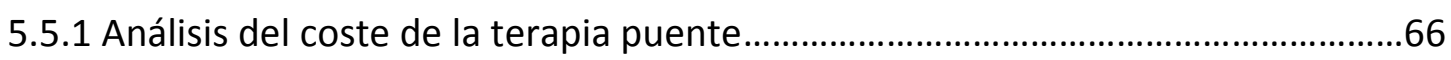

5.5.2 Análisis del coste de las complicaciones..................................................................66

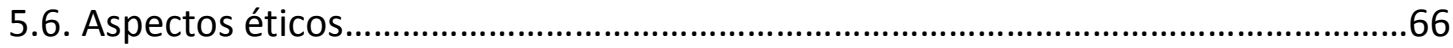

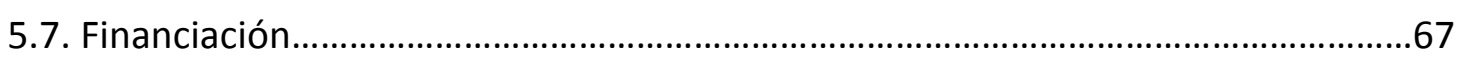

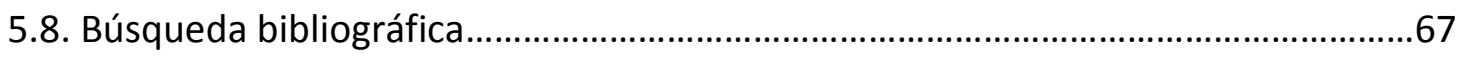

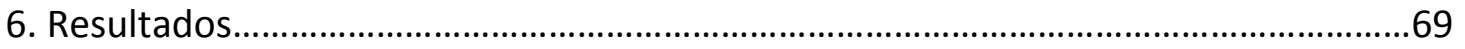

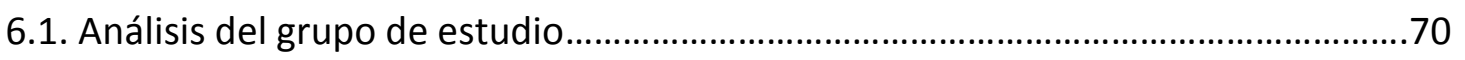

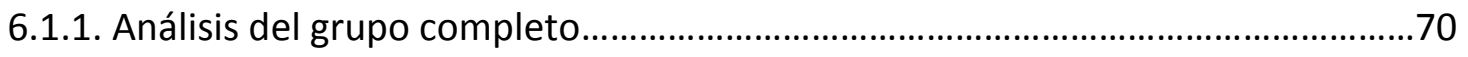

6.1.2. Grupo con diagnóstico de FA y bajo riesgo..........................................................71

6.2. Complicaciones hemorrágicas o trombóticas..........................................................73

6.2.1. Análisis de las complicaciones hemorrágicas..........................................................73

6.2.2. Análisis de las complicaciones trombóticas..........................................................74

6.3. Análisis de efectos secundarios por el uso de HBPM................................................75

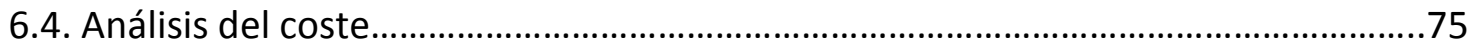

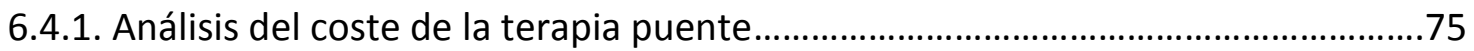

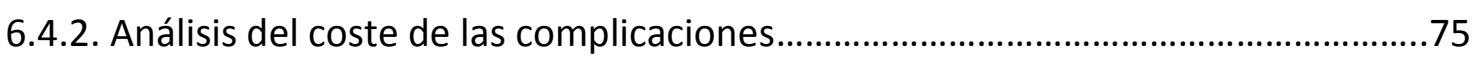

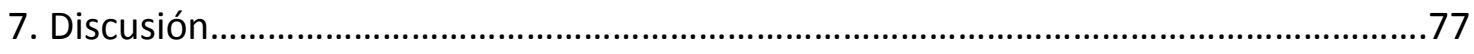

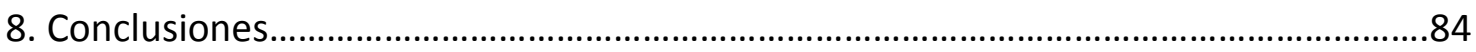

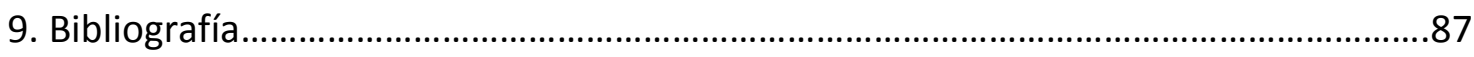


1. JUSTIFICACIÓN 



\section{JUSTIFICACIÓN}

La aplicación de las guías recomendadas por las sociedades científicas pretende mejorar el cuidado del paciente y minimizar los riesgos asociados a tratamientos farmacológicos o a los procedimientos invasivos.

Las guías están elaboradas por expertos y sustentadas habitualmente en estudios de medicina basada en la evidencia. Esto permite emitir unas recomendaciones internacionales que debieran ser seguidas en la práctica clínica.

Sin embargo, en muchas ocasiones, la implementación de estas guías no se efectúa o se dilata en el tiempo pudiendo generar en los pacientes complicaciones graves que provocan que el paciente deba ser atendido en el servicio de Urgencias, que condicionen el ingreso hospitalario y en alguna ocasión, pueden llegar a causar la muerte del paciente.

El análisis del seguimiento de las guías permite hacer una valoración de cuál es su cumplimiento y de la necesidad de introducir cambios en los protocolos locales.

Las recomendaciones del American College of Chest Physicians en su última edición proponen que en pacientes en tratamiento anticoagulante oral con fármacos antivitamina $\mathrm{k}$ que van a ser sometidos a un procedimiento invasivo programado, una pauta diferente en cuanto a la necesidad de sustituir el anticoagulante oral por heparina de bajo peso molecular según el grupo de riesgo (1).

Así, los pacientes se clasificarían según el grupo de riesgo de complicaciones tromboembólicas y en base a esta clasificación deberían recibir terapia puente con heparina de bajo peso molecular o no recibir ningún tipo de fármaco anticoagulante.

Por tanto, los pacientes quedarían diferenciados en 2 grupos:

En pacientes portadores de válvula cardíaca mecánica, fibrilación auricular o tromboembolismo venoso con alto riesgo de tromboembolismo, se sugiere realizar terapia puente durante la interrupción del anticoagulante antivitamina k. 
En pacientes de bajo riesgo, se sugiere no realizar terapia puente.

En el estudio que vamos a detallar se hace un análisis de la implementación correcta de estas guías y se recogen las complicaciones que han surgido relacionadas con el seguimiento o no de las mismas.

Así mismo, se hace un análisis de los costes farmacológicos en relación al uso de terapia puente y de los costes derivados de la atención de las complicaciones generadas. 
2. INTRODUCCIÓN 


\section{INTRODUCCIÓN}

\subsection{INTRODUCCIÓN AL DESCUBRIMIENTO DE LOS ANTICOAGULANTES ORALES ANTAGONISTAS DE LA VITAMINA K}

El tratamiento anticoagulante oral con fármacos antivitamina $\mathrm{K}$, se basa en la administración de los fármacos llamados cumarínicos, derivados de la 4hidroxicumarina y de las indanedionas, moléculas derivadas de la indan-1:3 diona.

El nacimiento de esta familia de medicamentos se debió al descubrimiento del dicumarol. Schofield, en 1922 (2) describe la llamada enfermedad del trébol dulce, que afectaba al ganado bovino y ovino de la región de Alberta en Canadá. Los veterinarios y ganaderos sabían que el ganado ocasionalmente y sin razón aparente sangraba en abundancia. Se trataba de una diátesis hemorrágica grave que podía conducir a la muerte de los animales al complicar procedimientos como la castración o la descornación. Este autor describió la existencia de una alteración en la coagulación de los animales afectados y observó que los animales que presentaban esta complicación, eran alimentados con trébol dulce mal curado.

Unos años más tarde Roderick (3) describe el mismo cuadro en el ganado de Dakota del Norte y observa una reducción del contenido de protrombina en la sangre de los animales afectos; así mismo, demuestra que la "fracción protrombínica" del plasma de los animales sanos corrige el defecto de coagulación de los animales enfermos.

Posteriormente Dam en 1935 describió que los pollos alimentados con una dieta desprovista de sustancias solubles en éter desarrollaban una tendencia hemorrágica que se curaba añadiendo alfalfa a la dieta o con la administración de una sustancia liposoluble que denominó vitamina K (4). 
Será en 1939, cuando Binkley et al sinteticen la sustancia contenida en la alfalfa y que llamarán vitamina K 1 o filoquinona (5).

Quick en 1936 describió el tiempo de protrombina y observó que este estaba prolongado en pollos alimentados con dietas carentes en sustancias liposolubles, similares a las empleadas por Dam y también cuando la alimentación fundamental de los animales se basaba en el trébol dulce deteriorado (6).

La sustancia presente en el trébol dulce mal conservado fue aislada por Link y llamada dicumarol; este es un compuesto que deriva de la cumarina presente en el trébol fresco (7).

Durante el proceso de fermentación del pienso, la cumarina primero se oxida, transformándose en 4-hidroxicumarina y luego, dos moléculas de esta reaccionando con una de formaldehido, dan lugar al dicumarol. Posteriormente se descubrió la similitud estructural del dicumarol con la vitamina $K$, describiéndose la existencia de un antagonismo reversible entre ambas sustancias (8).

La forma activa de la vitamina $\mathrm{K}$ (hidroquinona) actúa como cofactor en la carboxilación de los residuos de ácido glutámico de las proteínas vitamina Kdependientes. Esta acción es realizada por una carboxilasa que a la vez, transforma a la vitamina en su epóxido. Esta, para volver a participar en el proceso de carboxilación, debe ser transformada, primero en quinona, por una epóxido-reductasa y posteriormente, en hidroquinona por quinona-reductasas. Los anticoagulantes orales vitamina $\mathrm{K}$ dependientes inhiben a la epóxido-reductasa y a un tipo de quinonasreductasas, lo que impide la recuperación de la vitamina $\mathrm{K}$ (Fig. 1) 
Residuo

Residuo

Ácido glutámico

Ácido carboxiglutámico

(Glu)

(Gla)

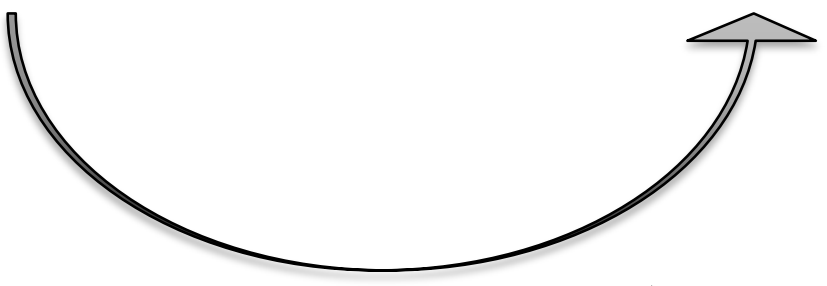

V.KH2

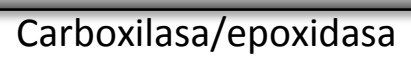

V.KO

Hidroquinona

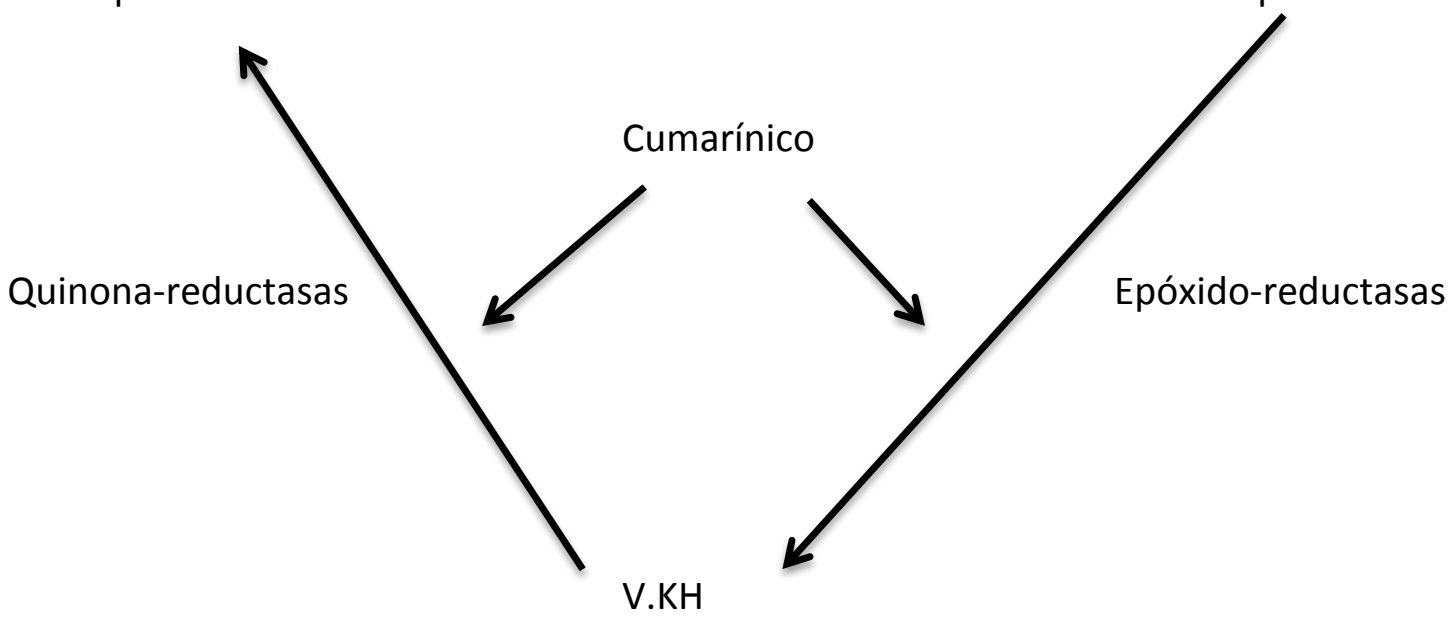

Quinona

Los residuos de ácido glutámico dicarboxilado (residuos Gla), presentes en las proteínas vitamina K-dependientes, son necesarios para el establecimiento de puentes de calcio $\left(\mathrm{Ca}^{++}\right)$con los fosfolípidos de la bicapa lipídica de la membrana activada de las plaquetas y de otras células, sobre las que tiene lugar, primero la activación del factor $X$ por el factor IXa, en presencia del F VIIla y luego, el paso de protrombina a trombina por acción del F Xa en presencia del F Va. Las proteínas que se sintetizan en ausencia de vitamina K o bajo la acción de los anticoagulantes orales, carecen de residuos Gla o poseen un número reducido de ellos, lo que impide su fijación a las membranas y su participación en la coagulación. 
En la tabla 1 se relacionan las principales proteínas vitamina K-dependientes, el número de residuos Gla que contienen y su vida media destacando la brevedad de las correspondientes a F VII y a la proteína C.

Tabla 1: Proteínas dependientes de la vitamina K.

Proteína $\quad$ Residuos Gla vida media (horas)

\begin{tabular}{|l|cc|}
\hline Protrombina & 10 & 60 \\
Factor VII & 10 & 6 \\
Factor IX & 12 & 24 \\
Factor X & 11 & 30 \\
Proteína C & 9 & 6 \\
Proteína S & 10 & 42 \\
\hline
\end{tabular}

\subsubsection{Uso de los anticoagulantes orales antagonistas de la vitamina $K$ en humanos}

El primer anticoagulante utilizado fue el dicumarol y Butt et al en 1941 trataron al primer paciente con este fármaco (9).

Antes en 1940, Link y Campbell dieron a conocer un "agente hemorrágico" que habían sintetizado $(10,11)$ al que llamaron dicumarol.

En 1940 Link que dirigía las investigaciones en animales, intuyó una conexión con la vitamina $\mathrm{K}$, demostrando que ésta revierte completamente los efectos del trébol mal curado y del dicumarol y asignó los derechos de la patente a los mecenas de su investigación, la Wisconsin Alumni Research Foundation que patentó el dicumarol en 1941. 
Encabezados por Allen, el grupo de la Clínica Mayo fue el primero en administrar dicumarol a seres humanos en mayo del mismo año (12).

Posteriormente, Link trabajó en la investigación del dicumarol como rodenticida y patentó en 1948 a favor de la Wisconsin Alumni Research Foundation un derivado del dicumarol con el nombre de warfarina (nombre que corresponde a las iniciales de la fundación más el sufijo "arina") (13).

En 1948 la warfarina fue promocionada como veneno para los roedores.

En 1951, un recluta de la marina intentó sin éxito suicidarse con 567 mg de warfarina. Su sorprendente recuperación completa induciría a Shapiro y Meyer a utilizar la warfarina en humanos, ensayándose esta en voluntarios en 1953 (14) y 1954 (15) observándose su superioridad en cuanto a manejo de los niveles de anticoagulación con respecto al dicumarol.

La warfarina se presentó comercialmente en 1954 y fue Pollock quien expuso las primeras experiencias clínicas en 1955 (16).

La warfarina y otros anticoagulantes orales antivitamina $\mathrm{K}$ comercializados actualmente son sintetizados químicamente.

\subsection{CLASIFICACIÓN DE LOS ANTICOAGULANTE ORALES ANTAGONISTAS DE LA VITAMINA K}

Los anticoagulantes orales antagonistas de la vitamina $K$ se dividen en 2 grandes grupos: los derivados cumarínicos y los derivados de la indanediona no utilizándose estos últimos en la práctica clínica (17). 
La duración de su acción es lo que diferencia a los derivados cumarínicos siendo los más utilizados los siguientes:

-Biscumacetato de etilo: de acción muy corta.

-Acenocumarol o nicumalone: tiene una vida media bastante corta. Es el anticoagulante más utilizado en España.

-Warfarina: tiene una acción más prolongada y es el más utilizado en el mundo.

-Femprocumon: de acción más prolongada que el anterior.

-Fluindiona: apenas se utiliza.

\subsubsection{Mecanismo de acción}

Los anticoagulantes orales antivitamina $K$, actúan impidiendo que la vitamina $K$ intervenga como cofactor en la reacción de carboxilación que tiene lugar en el hígado y mediante la cual los factores de coagulación del complejo protrombínico (factores II, VII, IX y X y los inhibidores de proteínas C y S) pasan de ser precursores inactivos a ser proteínas activas. (Fig 2).

Fig 2: Ciclo de la vitamina K y reacción de carboxilación

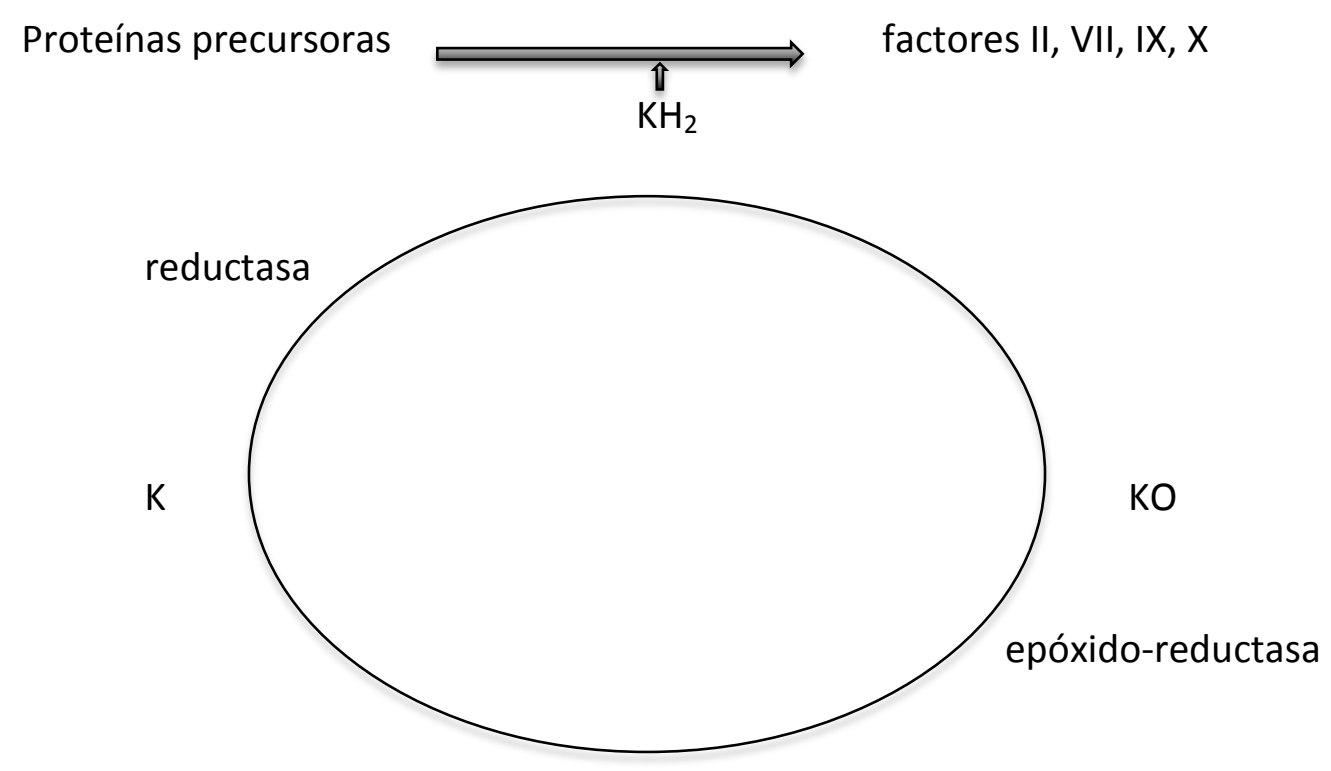


Los fármacos antagonistas de la vitamina $\mathrm{K}$ ( $\mathrm{AVK}$ ) bloquean el ciclo de la vitamina $\mathrm{K}$ de tal forma que no puede intervenir en la reacción de carboxilación, neutralizando por una parte la reductasa responsable del paso de vitamina $\mathrm{K}$ epóxido (KO) a vitamina $\mathrm{K}$ quinona (K), y de otra parte otra reductasa responsable del paso de vitamina $\mathrm{K}$ a vitamina $\mathrm{K}$ reducida $(\mathrm{KH} 2)$.

Los cumarínicos que poseen una similitud estructural con la vitamina $\mathrm{K}$, interfieren de forma competitiva en el proceso de regeneración de esta. En ausencia de vitamina activa, se sintetizan proteínas carentes en residuos Gla (ácido carboxiglutámico) y por tanto, incapaces de participar en la coagulación (18).

La vitamina $\mathrm{K}$ es una vitamina liposoluble que se puede adquirir por dos vías:

-vía exógena sobre todo con la ingesta de vegetales verdes: es la vitamina $\mathrm{K}_{1} \mathrm{O}$ filoquinona, activa al $100 \%$.

-vía endógena procedente de la síntesis realizada por las bacterias de la microbiota intestinal (como Escherichia Coli): es la menaquinona o vitamina $K_{3}$, activa al $75 \%$.

Los requerimientos diarios de vitamina $\mathrm{K}$ son muy bajos, entre 0,7 y $1,4 \mathrm{mcg} / \mathrm{kg}$.

Tras la administración por vía oral del anticoagulante, este se absorbe completamente en la primera porción del intestino delgado, pasa a la sangre fijándose en una proporción del 97 \% a las proteínas del plasma, es metabolizado en el hígado y eliminado fundamentalmente por la orina.

La acción terapéutica de los AVK depende de la concentración del fármaco libre en plasma, descendiendo los factores del complejo protrombínico de forma progresiva y dependiendo de la vida media de cada uno de ellos, no alcanzándose el efecto 
antitrombótico hasta pasados 3 o 5 días de iniciado el tratamiento según el AVK utilizado (19).

\subsubsection{Antídoto}

Los AVK poseen un antídoto que es la vitamina $\mathrm{K}$, con facilidad para su uso puesto que puede utilizarse tanto por vía oral como intravenosa (20).

Así mismo, el plasma y los concentrados de factores del complejo protrombínico inyectados vía intravenosa, pueden neutralizar el efecto de los AVK (21)

\subsection{ACENOCUMAROL}

Dentro de los fármacos $A V K$, nos centraremos en el acenocumarol que es el AVK más utilizado en nuestro medio.

De las características del fármaco destaca su biodisponibilidad, > $60 \%$ y que presenta una vida media de entre 8 a 11 horas (22).

El acenocumarol alcanza su acción terapéutica (esto es, la reducción de la concentración de factores de la coagulación), en general, a las 48 o 72 horas de haberse iniciado su administración, y su efecto desaparece también muy rápidamente tras su suspensión, lo que le confiere una gran comodidad de manejo (23); habiendo sido demostrada su utilidad clínica por Johnson et al (24). 


\subsection{CONTROL ANALÍTICO DE LOS ANTICOAGULANTES ORALES ANTAGONISTAS DE LA VITAMINA K}

Cuando el uso de AVK se generalizó, quedó patente que el tratamiento anticoagulante oral precisaba de un cuidadoso control analítico, debido a la gran variabilidad individual en la respuesta al fármaco; se observó que esta respuesta no

era predecible por el peso, por la edad o por otros factores y que determinadas circunstancias como la alimentación, la interacción con fármacos y otras, podían suponer un cambio importante en los valores anticoagulantes.

Además, se determinó que para ser eficaz se requería un estrecho margen terapéutico, condicionando este intervalo el tratamiento con dosis ineficaces (si no se alcanza el nivel de anticoagulación requerido para una determinada patología), adecuadas o excesivas (si la dosis de AVK provoca una hiperanticoagulación).

Esta inquietud llevó a que se iniciaran estudios para determinar cuál sería la mejor prueba analítica para el control de estos fármacos.

El tiempo de protrombina (TP) que en la década de los 40 del siglo $X X$, se consideraba que medía solo la actividad de la protrombina o sea del factor II de la coagulación (FII), se mostró como la prueba ideal (25).

Allen et al. en 1942 propusieron como nivel terapéutico el comprendido entre la razón paciente/control $(\mathrm{P} / \mathrm{C})$ de 1.5 a 3 y Quick, posteriormente consideró como valores óptimos los comprendidos entre 2 y 2.5 ; será este último el intervalo terapéutico aceptado finalmente por la Sociedad de Cardiología Americana (26).

Sin embargo, estos márgenes terapéuticos se basaban en resultados del TP obtenidos mediante el uso de un reactivo con tromboplastina de conejo de muy baja 
sensibilidad. Esto suponía que el uso de las diferentes tromboplastinas utilizadas para los estudios de coagulación condicionaran una gran variabilidad en los resultados (27).

Pronto se hizo patente la necesidad de un modelo matemático que permitiera obtener resultados equivalentes con reactivos de diferente sensibilidad. En 1967 Biggs y Denson propusieron un primer modelo, basado en la regresión lineal entre razones $\mathrm{P} / \mathrm{C}$ (paciente/control). Sin embargo, los resultados no fueron satisfactorios (28).

Por ello en 1981, el Bureau of Reference de la Comunidad Económica Europea, mediante un trabajo cooperativo, estableció un segundo modelo, basado en la regresión lineal de los logaritmos de los tiempos en segundos. Así, enfrentando la tromboplastina de referencia (ordenadas) a la preparación problema (abscisas), la sensibilidad de esta venía determinada por la pendiente de la línea de regresión, que se denominó Índice de Sensibilidad Internacional (ISI). Elevando la razón P/C al valor ISI se hallaría la razón teórica que se hubiera obtenido de realizar la prueba con el primer estándar humano. A este valor se le denomina Razón Normalizada Internacional (INR) y es la única forma correcta de expresar los resultados del TP cuando corresponden al control del tratamiento anticoagulante oral (29).

$$
\text { INR = (TP paciente/TP control })^{|S|}
$$

Cada tromboplastina se calibra según la de referencia de la Organización Mundial de la Salud (OMS) a la que se asignó un ISI de 1. Así, los resultados del INR pueden ser uniformes al utilizar el ISI como factor de corrección (30).

Por tanto, el uso del INR permite (31):

- Minimizar la variabilidad entre laboratorios, reactivos y coagulómetros.

- Facilitar el acuerdo internacional entre rangos terapéuticos y permitir la comparación directa de ensayos clínicos.

- Mayor uniformidad en el manejo del tratamiento anticoagulante oral (TAO). 
- Mejoría de los resultados en los pacientes, al permitir una dosificación del tratamiento que depende menos de la variabilidad en la sensibilidad del reactivo de tromboplastina.

\subsubsection{Control analítico por punción venosa.}

Los AVK son bien tolerados, con una relación coste-eficacia elevada, con una experiencia de uso difícil de igualar, con un coste muy bajo y un porcentaje de control, en la vida real razonable, alcanzando el rango de control excelente en aquellos pacientes que realizan el autocontrol.

El control facilita la cumplimentación y si hay una mala adherencia al tratamiento anticoagulante oral se puede detectar precozmente dado que los controles son periódicos y frecuentes.

Durante muchos años del control del TAO se realizaba obteniendo una muestra de sangre total por punción venosa en la fosa cubital del antebrazo y analizando posteriormente la muestra en el coagulómetro convencional obteniendo el tiempo de protrombina junto con el INR.

\subsubsection{Control analítico por punción digital}

El uso generalizado del tratamiento anticoagulante oral y el mayor número de pacientes con indicación para este tratamiento llevó al desarrollo de coagulómetros portátiles (32).

Las dificultades de acceso al centro hospitalario hicieron que estos sistemas de medida del INR se extendieran y permitieran la descentralización del control del tratamiento anticoagulante oral mejorando la calidad de vida de los pacientes al eliminar la obligatoriedad del desplazamiento del paciente hasta el centro hospitalario ya que el control del TAO podía realizarse por punción digital en su Centro de Salud. 
Los coagulómetros portátiles son cómodos y fáciles de usar y determinan el valor INR a partir de una gota de sangre total capilar habiendo probado que son precisos y fiables (33).

Además, se demostró que esta solución era coste-efectiva (34).

Hay diversos modelos en el mercado, pero nos centraremos en el coagulómetro CoaguChek $^{\circledR} \mathrm{XS}$ que es el utilizado en nuestro medio.

\subsubsection{Coagulómetros portátiles: CoaguChek $^{\circledR} \mathrm{XS}$}

El sistema CoaguChek ${ }^{\circledR}$ XS puede utilizarse en cualquier momento o lugar y la determinación de INR puede realizarse en el Hospital, Centro de Salud o domicilio del paciente (35).

Los resultados son rápidos obteniéndose un INR exacto en un minuto y fiables, ya que realiza comprobaciones automáticas de control de calidad puesto que lleva un sistema incorporado en cada tira reactiva.

Este coagulómetro ha demostrado su exactitud en los resultados y una precisión comparables a la obtenida en el laboratorio. Así, en el meta-análisis efectuado por Caballero-Villarraso et al, se observó una alta correlación entre los coagulómetros portátiles y los coagulómetros convencionales en cuanto a validez analítica y por tanto, eficacia equivalente (36).

\subsubsection{Coste del control con coagulómetros portátiles}

Aunque hay pocos artículos que incluyan la evaluación económica, los resultados sugieren una mejor relación costo-beneficio de los coagulómetros portátiles en comparación con los coagulómetros convencionales $(36,37,38)$. 


\subsubsection{Preferencias de los pacientes}

La mayoría de los pacientes prefiere que se les extraiga una pequeña gota de sangre (solo $8 \mu \mathrm{L}$ ) mediante punción en el dedo a la extracción de sangre venosa en la fosa cubital del brazo (39).

\subsubsection{Ventajas del uso del coagulómetro portátil}

Se ha demostrado que se consiguen mejores resultados clínicos en los pacientes pues la facilidad de acceso permite aumentar la frecuencia de las pruebas y esto puede reducir la aparición de eventos fatales (40).

Aunque no ha sido materia de investigación habitual la supervivencia de los pacientes en TAO controlados con coagulómetros portátiles, sí que se ha visto una menor mortalidad en este grupo en los estudios que analizan este dato (36).

Otra de las ventajas de los coagulómetros portátiles ha sido que estos sistemas de lectura rápida del INR han permitido el autocontrol por los propios pacientes tras un entrenamiento previo por el personal sanitario de los Servicios de Hematología y con apoyo de los mismos en la toma de decisiones (41), facilitando el correcto seguimiento del tratamiento anticoagulante oral y suponiendo una gran ventaja y comodidad, fundamentalmente en pacientes jóvenes.

Se ha observado que los pacientes en TAO con autocontrol con coagulómetros portátiles, tienen mejores resultados de medición analítica y menores tasas de eventos tromboembólicos (36).

Las ventajas del autocontrol de la anticoagulación incluyen una mejora de la calidad de vida debido a una reducción de los desplazamientos del paciente al centro de salud y a un mejor control de la anticoagulación puesto que se reducen las cifras fuera del rango terapéutico ya que las mediciones de INR se realizan con más 
frecuencia. Por lo tanto, el autocontrol de la anticoagulación tiene el potencial de reducir la incidencia de episodios tromboembólicos y de sangrado en pacientes que reciben terapia anticoagulante oral a largo plazo (38).

\subsection{DESCENTRALIZACIÓN DEL CONTROL DEL TRATAMIENTO ANTICOAGULANTE ORAL}

La aparición de coagulómetros portátiles y el desarrollo tanto de las Unidades de Coagulación dentro de los Servicios de Hematología como de la propia Atención Primaria propiciaron que actualmente la mayoría de los pacientes anticoagulados con fármacos antagonistas de la vitamina $\mathrm{K}$ sean controlados a través de estos dispositivos en Atención Primaria.

\subsubsection{Programas para la gestión del tratamiento anticoagulante oral}

Paralelamente a la aparición de coagulómetros portátiles se diseñaron programas informáticos que han permitido la conexión vía página web entre el Hospital y el Centro de Salud.

En el mercado hay numerosos programas de software que permiten la gestión del TAO tanto de los pacientes ambulatorios como de los pacientes ingresados en el hospital.

Esto ha facilitado la interacción entre ambos ámbitos pues estos programas permiten la inclusión desde el Centro de Salud de datos clínicos junto con el INR, obtenido y enviado en tiempo real. Así, cualquier médico con acceso al programa puede realizar una validación clínica pautando la dosis del AVK y automáticamente se envía el informe en segundos (42).

La descentralización del TAO supone una nueva organización de la Sección de Coagulación dentro de los Servicios de Hematología y ofrece una serie de ventajas como son las siguientes: 
- Comunicación directa con los Centros de Salud.

- Aumento de la satisfacción del paciente y de su calidad de vida.

- Actualización continúa del registro del TAO del paciente.

- Tiempo ganado por el personal sanitario.

- Minimización de costes.

\subsection{CONTROL TERAPÉUTICO DE LOS ANTICOAGULANTES ORALES ANTAGONISTAS DE LA VITAMINA K}

\subsubsection{Unidad de control del tratamiento anticoagulante oral}

Los objetivos de una Unidad de control del tratamiento anticoagulante oral (TAO) son la calidad técnica en la fase analítica y la calidad en la atención al paciente.

\subsubsection{Calidad técnica de la fase analítica}

El concepto de calidad técnica incluye una correcta selección de los reactivos y de los coagulómetros. Los centros hospitalarios cuentan con coagulómetros automatizados que proporcionan resultados muy precisos y gran reproducibilidad.

La garantía de la calidad vendrá avalada por sistemas para su control interno y externo; así cada centro estará inscrito en un programa de garantía de la calidad, pudiendo ser de ámbito local, nacional o internacional (43).

\subsubsection{Calidad en la atención al paciente}

El segundo aspecto en la gestión de una unidad de control del TAO, es el que se refiere a la calidad en la atención al paciente.

Cuando un paciente debe iniciar el tratamiento anticoagulante oral debemos explicarle el objetivo de este tratamiento, la necesidad de una regularidad rigurosa en 
la toma del fármaco y en la asistencia a los controles periódicos, así como de los efectos secundarios y de los riesgos de sangrado (en caso de anticoagulación excesiva) o complicaciones trombóticas (en caso de insuficiente anticoagulación).

Es conveniente disponer de un folleto en el que se expliquen las características de este tratamiento y se informe sobre situaciones que puedan modificar el mismo (embarazo, cambios bruscos en el régimen alimentario u otros), así como un listado de fármacos potenciadores e inhibidores.

La atención al usuario incluirá en cada control, la existencia de una entrevista con el personal de enfermería idealmente en un ambiente privado, de modo que el paciente pueda exponer libremente sus dudas.

La nueva dosis se entregará en un informe lo suficientemente completo como para permitir la visita en otro centro, en caso de viaje, o en el área de Urgencias. Actualmente los sistemas informáticos permiten la visualización de los informes emitidos por el médico dentro de un área de salud e incluso dentro de una comunidad autónoma.

En dicho informe deben constar los datos personales del paciente, el valor del INR en el último control, el anticoagulante utilizado, la dosificación hasta el próximo control, expresada claramente y desglosada en forma de calendario para una más fácil comprensión.

Debe constar un breve resumen de los últimos controles con sus valores de INR y las dosis que se prescribieron y así facilitar al médico prescriptor la tarea de pautar la siguiente dosis.

El paciente es atendido por el personal de enfermería que mientras realiza la punción digital le someterá a una breve entrevista preguntándole sobre los cambios más relevantes; posteriormente, el valor del INR proporcionado por el coagulómetro 
será introducido en el programa informático y más tarde, el médico emitirá un informe o calendario de dosificación.

Se trata de un sistema ágil que permite atender al paciente anticoagulado en un espacio de tiempo relativamente breve. La descentralización del control del TAO ha terminado con la masificación del número de pacientes que debían acudir al hospital para el control.

La descentralización total o parcial reduce la posibilidad de comunicación entre el paciente y el médico pasando a tomar un papel preponderante el personal de enfermería.

\subsection{CONTROL DE LOS ANTICOAGULANTE ORALES ANTAGONISTAS DE LA VITAMINA K}

En relación al manejo de los cumarínicos existe una recomendación internacional de no iniciar su administración con una dosis de carga, ya que ello no acorta de modo sustancial el tiempo necesario para alcanzar el nivel terapéutico y aumenta el riesgo de aparición de necrosis cutánea trombótica en pacientes con déficit de proteína $C$ (debido a la corta vida media de esta proteína) y también la posibilidad de complicaciones hemorrágicas en los pacientes más sensibles.

También hay que tener en cuenta que no todos los fármacos anticoagulantes tienen el mismo tiempo de respuesta ni de eliminación del efecto anticoagulante una vez suprimida su administración.

Desde la introducción de estos fármacos se consideró deseable que tuvieran un corto período de inducción de su efecto y también de eliminación tras su suspensión, pero a la vez una acción lo suficientemente prolongada para permitir una anticoagulación homogénea sin oscilaciones con una sola dosis diaria. 
Estas condiciones las cumple el acenocumarol que es el anticoagulante oral más introducido en nuestro país.

\subsubsection{Control terapéutico de los anticoagulante orales antagonistas de la vitamina $K$}

El control terapéutico se define como la proporción de tiempo que un paciente permanece anticoagulado dentro del rango terapéutico definido para su patología, expresado como ratio normalizado internacional (INR).

Varios factores influyen en el control como por ejemplo, la calidad del laboratorio, la experiencia del que pauta la dosis y el nivel de conocimientos sobre anticoagulación del paciente.

En la mayoría de los países de nuestro entorno los Servicios de Hematología han constituido Unidades de control del tratamiento anticoagulante oral con personal específico adiestrado y en los cuales el personal de enfermería juega un papel destacado.

El personal de enfermería recibe al paciente y mientras practica la punción digital realiza un breve interrogatorio al paciente acerca de las posibles complicaciones hemorrágicas del tratamiento, cambios en la medicación, enfermedades intercurrentes y o procedimientos quirúrgicos o invasivos a los que debe someterse.

Una vez obtenido el resultado del INR, este es introducido en el programa informático que permite la dosificación y la elaboración de un informe detallado de la dosis diaria.

Para valorar en un paciente si el control terapéutico es el adecuado, Rosendaal et al. propusieron un método en el que se calcula el tiempo personal específico de INR incorporando la frecuencia de las mediciones de INR y sus valores reales, y suponiendo que los cambios entre mediciones de INR consecutivas son lineales en el tiempo. 
El tiempo en rango terapéutico (TRT) calculado mediante la fórmula de Rosendaal refleja la calidad de la anticoagulación de los pacientes en tratamiento con AVK, que es correcto si es > del $65 \%$ (44).

El inconveniente de que el paciente no se encuentre dentro del rango terapéutico establecido para su patología es el riesgo de eventos trombóticos cuando se encuentra infraanticoagulado o de eventos hemorrágicos, si presenta una excesiva anticoagulación.

Se consideró que un paciente presentaba un INR lábil cuando el tiempo en rango terapéutico era $<$ del $60 \%(45)$.

Pisters et al. en 2010 observaron que no existía una herramienta útil para cuantificar el riesgo de sangrado asociado al TAO en pacientes con fibrilación auricular (FA), y plantearon un estudio cuyo objetivo fue desarrollar una puntuación de riesgo "práctica", que permitiera estimar el riesgo en 1 año de hemorragia grave (definida como hemorragia intracraneal, que provoca hospitalización, o casusa una disminución de hemoglobina $>2 \mathrm{~g} / \mathrm{L}$ y / o requiere transfusión).

Este estudio se basó en la encuesta Euro del corazón sobre la FA que incluyó a 3.978 pacientes en los que se realizó un seguimiento completo de todos los factores de riesgo de sangrado; posteriormente, dichos factores fueron usados en un análisis multivariante asociados a factores de riesgo de sangrado históricos.

Toda la información obtenida permitió elaborar la "escala HAS-BLED", que valora el riesgo de sangrado y que incluye los siguientes factores: hipertensión, función renal / hepática anormal, accidente cerebrovascular, historial de sangrado o predisposición, cociente normalizado internacional lábil, ancianos (> 65 años), drogas / alcohol (concomitantemente). 
En el grupo de estudio se produjeron 53 hemorragias importantes (1,5\%) durante el seguimiento de 1 año. Se objetivó que la tasa de sangrado anual aumentaba cuando el paciente presentaba un mayor número de factores de riesgo. La precisión predictiva en la población general utilizando estos factores de riesgo fue consistente cuando se aplicó en diferentes subgrupos.

Esta sencilla escala de valoración del riesgo de hemorragia (HAS-BLED) proporciona una herramienta práctica para evaluar el riesgo de hemorragia individual de los pacientes con FA.

\subsection{INDICACIONES DEL TRATAMIENTO ANTICOAGULANTE ORAL}

Son varias las indicaciones del TAO pero en nuestro estudio nos centraremos en la FA.

\subsubsection{Fibrilación auricular}

\subsubsection{Epidemiología}

La FA es la arritmia más frecuente y la que genera mayor número de consultas a los Servicios de Urgencias, así como más días de ingreso hospitalario (46).

La FA no solo se asocia a un incremento de la morbilidad (47) sino también de la mortalidad (48).

\subsubsection{Prevalencia}

La prevalencia de la FA se incrementa con la edad, tanto en varones como en mujeres y aunque en un porcentaje de pacientes se asocia con enfermedad cardíaca orgánica, en un gran número de ellos la aparición de FA se detecta sin patología cardíaca establecida. 
Se estima que la prevalencia de FA es de aproximadamente el $2 \%$ de la población general, cifra que aumenta con la edad y con el aumento de comorbilidades (49).

Debido al aumento de la esperanza de vida, las previsiones indican que la prevalencia de la FA se duplicará en los próximos 50 años, pudiendo variar esta

predicción según el grado de control de los factores de riesgo cardiovascular, especialmente de la hipertensión arterial y de la obesidad (50).

Por tanto, la presencia de la FA está en relación con la existencia de factores de riesgo ligados al estilo de vida como obesidad, sedentarismo, hipertensión, diabetes mellitus y síndrome de apneas/hipoapneas del sueño.

En los países desarrollados, donde ambos factores (envejecimiento de la población y factores ligados al estilo de vida) convergen, se puede vaticinar que la FA llegará a tener cotas epidémicas en los próximos años (51).

El incremento de la prevalencia en relación a la edad está ampliamente constatado (52).

En los estudios europeos, la prevalencia en la población general se estima entre el 0,2 y el $2 \%$ y, en poblaciones mayores de 50 años se ha estimado entre el 1,5 y el 5,5\%. El estudio Rotterdam analizó la prevalencia de la FA en una población mayor de 55 años, siendo del 5,5\% de la población total, del 0,7 \% en el grupo entre 55-59 años y del $17,8 \%$ en el grupo de 85 o más años (53).

En España se han realizado varios estudios dirigidos a conocer la situación de la FA. En el estudio VAL-FAAP que analizó a cerca de 120.000 sujetos asistidos en las consultas de Atención Primaria, la proporción de sujetos con FA alcanzó el 6,1\% (54). 
En la población general mayor de 40 años, la prevalencia de FA en España fue del $4,4 \%(55)$.

Cuando se asocia un factor de riesgo como es la hipertensión arterial el porcentaje de pacientes con FA se incrementa; así, se ha detectado FA en el 10,3\% de los pacientes hipertensos de 65 o más años (56).

\subsubsection{Incidencia}

El estudio Framingham de 1982 fue el primero que publicó una incidencia general de FA crónica del $2 \%$ entre los pacientes de 32 a 65 años a los que habían realizado un seguimiento de 22 años y efectuado un electrocardiograma cada 2 años (57).

En Europa según el estudio de Rotterdam realizado en población mayor de 55 años, la incidencia total fue de 9,9/1.000 personas/año, mayor en varones que en mujeres. La incidencia en el grupo de edad de 55-59 años fue de 1,1/1.000 personas/año, y llegaba a 20,7/1.000 personas/año en la franja de 80-84 años (53).

\subsubsection{Riesgo trombótico}

Las alteraciones hemodinámicas provocadas por la FA comportan un aumento significativo del riesgo de embolias y en particular de accidente cerebro vascular (ACV), siendo provocadas por la formación de trombos dentro de la aurícula izquierda (58).

La presencia de FA aumenta alrededor de cuatro veces el riesgo de ACV; el riesgo absoluto es del > $5 \%$ anual y varía desde menos del $1 \%$ hasta más del $12 \%$ en función de la presencia de determinados factores de riesgo (59).

El ictus asociado a FA tiene mayor mortalidad, un riesgo incrementado de recurrencias y además genera mayores secuelas (60) 
La FA también se asocia con un incremento del riesgo de muerte (61). En comparación con los pacientes sin FA, los sujetos con FA tienen el doble de riesgo de muerte (62).

\subsubsection{Tratamiento anticoagulante oral en Fibrilación Auricular}

La anticoagulación oral crónica está indicada para la mayoría de los sujetos con FA para prevenir complicaciones tromboembólicas (54).

Los antagonistas de la vitamina $\mathrm{K}(\mathrm{AVK})$ se han utilizado ampliamente durante décadas para este fin, dado que son capaces de reducir aproximadamente un $64 \%$ el riesgo de ictus y un $26 \%$ la mortalidad en comparación con placebo (63).

Varios estudios han demostrado la superioridad de warfarina frente a aspirina y placebo; en el estudio de Petersen et al, se observó que la incidencia de complicaciones tromboembólicas y la mortalidad vascular fue significativamente menor en el grupo tratado con warfarina (64).

Posteriormente en otro estudio con los mismos comparadores: warfarina, placebo y aspirina, que incluyó 1.244 pacientes, se observó una tasa de eventos del 1.6 $\%$ por año en los pacientes que se incluyeron en los brazos de tratamiento con warfarina y aspirina y, del 8.3 \% por año en los pacientes que recibieron de placebo.

Se demostró que el tratamiento antitrombótico con warfarina es eficaz para reducir el riesgo de accidente cerebrovascular y embolia sistémica en pacientes con FA (65). 


\subsection{EVALUACIÓN DEL RIESGO TROMBOEMBÓLICO}

\subsubsection{Escala $\mathrm{CHADS}_{2}$}

Dado que el riesgo embólico y hemorrágico no es homogéneo en todos los pacientes, es fundamental hacer una evaluación individual previa al inicio del tratamiento anticoagulante oral es decir, ponderar el beneficio de la reducción del riesgo tromboembólico frente al aumento del riesgo hemorrágico de los pacientes con FA.

Hay un gran número de factores que se asocian con el riesgo tromboembólico de los pacientes con FA por lo que la combinación de estos factores de riesgo se ha utilizado para diseñar escalas de estratificación de riesgo con el objetivo de identificar a los pacientes candidatos a recibir tratamiento anticoagulante oral(66).

La escala de riesgo $\mathrm{CHADS}_{2}$ es una de las más simples y ha sido usada para guiar la terapia antitrombótica desde que fue propuesta y validada en 2001 gracias a los estudios AFI (67) y SPAF (68).

En el estudio de Gage BF et al, los autores evaluaron el valor predictivo de los esquemas de clasificación que estiman el riesgo de accidente cerebrovascular en pacientes con FA. Había 2 esquemas de clasificación existentes en ese momento que se combinaron en el nuevo esquema "el índice $\mathrm{CHADS}_{2}$ " y se validaron los 3 esquemas de clasificación.

El esquema $\mathrm{CHADS}_{2}$ se formó asignando 1 punto a cada uno de los siguientes hallazgos, presencia de insuficiencia cardíaca congestiva, hipertensión, 75 años de edad o más, y diabetes mellitus y asignando 2 puntos por antecedente de accidente cerebrovascular o ataque isquémico transitorio. 
Se realizó un seguimiento de los pacientes que ingresaron por ACV y analizaron la utilidad de los 3 esquemas; tanto el esquema desarrollado por los Investigadores de Fibrilación Auricular (AFI) y el esquema de Prevención de accidentes cerebrovasculares en la FA III (SPAF) predijeron el riesgo de accidente cerebrovascular. Sin embargo, el índice $\mathrm{CHADS}_{2}$ fue el predictor más preciso (68).

Este esquema permite clasificar artificialmente el riesgo trombótico en bajo $\left(\mathrm{CHADS}_{2}=0\right)$, moderado $\left(\mathrm{CHADS}_{2}=1\right)$, y alto $\left(\mathrm{CHADS}_{2} \geq 2\right)(69)$.

Sin embargo, la escala CHADS $_{2}$ presentaba dos inconvenientes: mientras clasifica bien a los pacientes de alto riesgo, genera un gran grupo de riesgo intermedio (> $60 \%$ ) y la puntuación $\mathrm{CHADS}_{2}=0$ no identifica realmente a los pacientes que tienen bajo riesgo trombótico, ya que los pacientes encuadrados en esta categoría presentan una tasa anual de riesgo trombótico $>3,2 \%(70)$.

Además no incluía otros factores de riesgo que favorecían la aparición de eventos trombóticos. Por todo ello, en 2009 el grupo de Birmingham propuso la nueva escala de riesgo $\mathrm{CHA}_{2} \mathrm{DS}_{2}$-VASc.

\subsubsection{Escala $\mathrm{CHA}_{2} \mathrm{DS}_{2}$-VASc}

$\mathrm{CHA}_{2} \mathrm{DS}_{2}$-VASc es el acrónimo de: insuficiencia cardíaca, hipertensión, edad $\geq$ 75 años [doble], diabetes mellitus, ictus [doble], enfermedad vascular, edad 65-74 y sexo femenino). Esta escala se elaboró con los datos de la población del estudio Euro Heart Survey on Atrial Fibrillation (71).

La escala $\mathrm{CHA}_{2} \mathrm{DS}_{2}$-VASc añade tres factores de riesgo a la anterior escala: la importancia de la edad, asignando 2 puntos a los pacientes con FA y edad > de 75 años y 1 punto si tiene entre 65-74 años; 1 punto a la presencia de enfermedad vascular (infarto de miocardio, enfermedad arterial periférica o placa aórtica complicada), y 
otro punto si el sexo es femenino (pero no puntúa si es el único factor de riesgo presente).

La escala $\mathrm{CHA}_{2} \mathrm{DS}_{2}$-VASc demostró que mejora la identificación de pacientes con verdadero bajo riesgo, ya que identifica a aquellos que no se benefician de iniciar terapia anticoagulante oral $(72,73)$.

Así en un estudio de validación del esquema $\mathrm{CHA}_{2} \mathrm{DS}{ }_{2}$-VASc en 4.670 pacientes, la escala reclasificó a los pacientes con $\mathrm{CHADS}_{2}=1$. Un $26 \%$ de los pacientes presentaban una puntuación $\mathrm{CHA}_{2} \mathrm{DS}_{2}$-VASc $=1$, con un riesgo anual de ictus del $0.9 \%$ y se reclasificó a un $74 \%$ de los pacientes con $\mathrm{CHADS}_{2}=1$ en una puntuación $\mathrm{CHA}_{2} \mathrm{DS}_{2^{-}}$ VASc $\geq 2$, con un riesgo anual de ictus o embolia sistémica del 2,1 \% (74).

\subsubsection{Nuevas escalas de riesgo}

Han surgido nuevas iniciativas para mejorar la estratificación del riesgo en cuanto a mortalidad, posibilidad de ictus y hemorragia en pacientes con fibrilación auricular; en este estudio prospectivo se vio que el rendimiento de la herramienta de riesgo GARFIELD-AF fue superior a $\mathrm{CHA}_{2} \mathrm{DS}_{2}$-VASc en la predicción de accidente cerebrovascular y mortalidad y, superior a HAS-BLED para el sangrado, en general y sobre todo en pacientes de menor riesgo.

La herramienta GARFIELD-AF que utiliza la regresión por pasos para el análisis, tiene la posibilidad de ser incorporada en los sistemas electrónicos de rutina y permite la evaluación simultánea de los accidentes cerebrovasculares isquémicos, la mortalidad y los riesgos de sangrado derivados del TAO (75). 


\subsection{EVALUACIÓN DEL RIESGO HEMORRÁGICO}

El incremento del riesgo de hemorragia acompaña al tratamiento con anticoagulantes orales. La incidencia anual de sangrado intracraneal asociado a anticoagulación aumenta de 0,8 a 44,4/100.000 habitantes (76).

Por ello es necesario conocer el riesgo hemorrágico individual de los pacientes antes de iniciar el tratamiento antitrombótico. Hay numerosos factores de riesgo que predisponen al sangrado, por lo que se han desarrollado varias escalas para evaluar el riesgo hemorrágico en estos pacientes.

\subsubsection{Escala HAS-BLED}

La terapia antitrombótica disminuye el riesgo de fenómenos tromboembólicos de los pacientes que padecen FA a expensas de elevar el riesgo hemorrágico, por lo que resulta crucial la evaluación individual del riesgo de los pacientes analizando el beneficio clínico neto de la terapia antitrombótica.

Se dispone de varias escalas de evaluación del riesgo hemorrágico que pueden ayudar en la toma de decisiones. La escala HAS-BLED es la más más empleada en la práctica clínica y es el acrónimo de:

- Hypertension (hipertensión): presión arterial sistólica > 160 mHg: 1 punto.

- Abnormal kidney and/or liver function (disfunción renal o hepática): diálisis crónica o trasplante renal o creatinina sérica $\geq 2 \mu \mathrm{mol} / \mathrm{L}$; enfermedad

hepática crónica o alteración bioquímica de bilirrubina $>2$ veces el límite alto de la normalidad, más alanina amino-transferasa/ aspartato aminotransferasa (ALT/AST) $>3$ veces el límite alto de la normalidad: 1 punto cada una. 
- Stroke (ictus): antecedente de ictus, 1 punto.

- Bleeding (sangrado): historia de sangrado o predisposición a la hemorragia, 1 punto.

- Labile INR (INR lábil): INR alto o inestable o $<60 \%$ del tiempo en rango terapéutico (TTR), 1 punto.

- Elderly (ancianos): edad > 65 años, 1 punto.

- Drugs and/or alcohol (fármacos y alcohol): toma concomitante de fármacos (antiinflamatorios no esteroideos o AAS) o abuso de alcohol, 1 punto cada uno.

Un elevado riesgo hemorrágico (HAS-BLED > 3) no contraindica la anticoagulación cuando está indicada, pero sí que se deben extremar las precauciones para evitar hemorragias.

Esta escala ha sido validada por la Euro Heart Survey in Atrial Fibrillation Cohort (77). Un paciente con una puntuación de 0-1 se clasifica como bajo riesgo; 2 , como de riesgo moderado, $y \geq 3$ como de alto riesgo.

El esquema HAS-BLED es el que presenta mayor poder predictivo de hemorragia intracraneal en comparación con otros esquemas de riesgo hemorrágico (78) y también está validado para predecir el riesgo hemorrágico durante la terapia puente (79).

La escala HAS-BLED es fácil de recordar y, sobre todo, identifica los factores modificables (por ejemplo, control de la presión arterial, suspensión del tratamiento concomitante con un antiagregante plaquetario) que pueden predecir el riesgo hemorrágico de los pacientes. 


\subsubsection{Otras escalas}

Otro esquema de evaluación de riesgo de sangrado desarrollado es la escala ATRIA, derivada del estudio ATRIA-AF (80).

Este esquema utiliza los siguientes factores de riesgo: anemia (hemoglobina < $13 \mathrm{gr} / \mathrm{dl}$ los varones y $12 \mathrm{gr} / \mathrm{dl}$ las mujeres, 3 puntos), enfermedad renal grave (filtrado glomerular estimado $<30 \mathrm{ml} / \mathrm{min} / 1,73 \mathrm{~m} 2,3$ puntos), edad $>75$ años (2 puntos), sangrado previo (1 punto) e hipertensión (1 punto). Una puntuación de 0-3 se define como bajo riesgo; 4, como riesgo intermedio, $y \geq 5$, como alto riesgo. No obstante, se ha puesto de manifiesto en varios estudios su pobre poder predictivo y su incapacidad para predecir el sangrado intracraneal en comparación con la escala HAS-BLED. En una población de 937 pacientes anticoagulados con acenocumarol, el esquema HAS-BLED presentó mayor poder predictivo $(C=0,68$ frente a $C=0,59 ; p=0,0359)$ en comparación con el esquema ATRIA, lo que refuerza el valor del sencillo esquema HAS-BLED (81).

Por todo lo expuesto, la escala HAS-BLED es la recomendada en la mayoría de las guías de práctica clínica para la evaluación del riesgo hemorrágico $(82,83,84)$.

\subsection{MANEJO DEL TRATAMIENTO ANTICOAGULANTE ORAL EN EL PERIPROCEDIMIENTO}

\subsubsection{Evaluación del riesgo periprocedimiento}

Para determinar si es necesario interrumpir el TAO, así como decidir si se realiza terapia puente, se deben valorar dos puntos clave:

-El tipo de intervención quirúrgica o exploración que se va a realizar y su riesgo hemorrágico

-El riesgo trombótico de la indicación por la que está anticoagulado el paciente. 
La toma de decisiones no es sencilla ya que existen situaciones en que los riesgos son moderados, y establecer el balance adecuado entre el riesgo trombótico y hemorrágico puede tener un cierto grado de dificultad (85).

Para tomar una decisión equilibrada se han clasificado las diferentes intervenciones en base a su riesgo hemorrágico (Tabla 2)

Tabla 2: Valoración del riesgo hemorrágico de la intervención.

\begin{tabular}{|c|c|c|}
\hline Bajo riesgo & Alto riesgo & Muy alto riesgo \\
\hline Intervenciones dentales & Resto de cirugía mayor & $\begin{array}{l}\text { Vasculares (aorta, by- } \\
\text { pass, coronaria, valvular) }\end{array}$ \\
\hline $\begin{array}{l}\text { Procedimientos } \\
\text { dermatológicos }\end{array}$ & Intervenciones de retina & Intracraneal o espinal \\
\hline $\begin{array}{l}\text { Cataratas con anestesia } \\
\text { tópica }\end{array}$ & $\begin{array}{l}\text { Biopsia de próstata y } \\
\text { riñón }\end{array}$ & $\begin{array}{l}\text { Intervenciones de } \\
\text { próstata y vejiga }\end{array}$ \\
\hline Infiltraciones & $\begin{array}{l}\text { Resección de pólipos de } \\
\text { colon sésiles }\end{array}$ & $\begin{array}{l}\text { Cirugía de procesos } \\
\text { tumorales }\end{array}$ \\
\hline Biopsia de médula ósea & & Amigdalectomía \\
\hline $\begin{array}{l}\text { Endoscopia con biopsia } \\
\text { de mucosa* }\end{array}$ & & $\begin{array}{l}\text { Cirugía ortopédica } \\
\text { mayor }\end{array}$ \\
\hline $\begin{array}{l}\text { Implantación de } \\
\text { marcapasos }\end{array}$ & & $\begin{array}{l}\text { Pacientes con riesgo } \\
\text { hemorrágico individual } \\
\text { especialmente elevado }\end{array}$ \\
\hline
\end{tabular}

*Aunque en las endoscopias altas y bajas el riesgo es bajo, se suele suspender el TAO ante la posibilidad de necesitar hacer una biopsia de capas profundas de la pared o polipectomía.

Así mismo, los pacientes se clasifican en diferentes grupos de riesgo de complicaciones trombóticas en el periprocedimiento según la patología por la que están en tratamiento anticoagulante oral. (Tabla 3) 
Tabla 3: Valoración del riesgo trombótico según la indicación del tratamiento anticoagulante oral ante la cirugía $u$ otros procedimientos invasivos. Índice $\mathrm{CHA}_{2} \mathrm{DS}_{2}-$ VASc y antecedentes trombogénicos.

\begin{tabular}{|c|c|c|c|}
\hline & Bajo & Moderado & Alto \\
\hline $\begin{array}{l}\text { Válvulas cardíacas } \\
\text { mecánicas }\end{array}$ & $\begin{array}{l}\text { Posición aórtica } \\
\text { bivalva sin } \\
\text { factores de riesgo } \\
\text { Bioprótesis en } \\
\text { ritmo sinusal }\end{array}$ & $\begin{array}{l}\text { Posición aórtica } \\
\text { bivalva con FA, } \\
\text { ACV/AIT previo, } \\
\text { DM, IC o edad > } \\
75 \text { años }\end{array}$ & $\begin{array}{l}\text { Posición mitral } \\
\text { Posición aórtica } \\
\text { (antiguas) }\end{array}$ \\
\hline FA & $\begin{array}{l}\mathrm{CHA}_{2} \mathrm{DS}_{2} \text {-VASc: } 1-4 \\
\text { sin ACV/AIT/EP } \\
\text { previo }\end{array}$ & $\begin{array}{l}\mathrm{CHA}_{2} \mathrm{DS}_{2}-\mathrm{VASc}: 5-6 \\
\mathrm{ACV} / \mathrm{AIT} / \mathrm{EP}>3 \\
\text { meses }\end{array}$ & $\begin{array}{l}\mathrm{CHA}_{2} \mathrm{DS}_{2}-\mathrm{VASc}: \geq 7 \\
\mathrm{ACV} / \mathrm{AIT} / \mathrm{EP}<3 \\
\text { meses } \\
\text { Valvulopatía } \\
\text { reumática mitral }\end{array}$ \\
\hline $\begin{array}{l}\text { Antecedente de } \\
\text { TEV o trombofilia } \\
\text { o stents }\end{array}$ & TEV $>12$ meses & $\begin{array}{l}\text { TEV 3-12 meses } \\
\text { Trombofilia no } \\
\text { grave } \\
\text { TEV recurrente } \\
\text { TEV + cáncer }\end{array}$ & $\begin{array}{l}\text { TEV reciente }(<3 \\
\text { meses) } \\
\text { Trombofilia grave } \\
\text { Stents coronarios }\end{array}$ \\
\hline
\end{tabular}

${ }^{a}$ En cirugía programada en pacientes con stents coronarios metálicos, retrasar la cirugía al menos 6 semanas desde la implantación, y en stents farmacoactivos, retrasarla al menos 6 meses.

ACV: accidente cerebrovascular; AIT: accidente isquémico transitorio; DM: diabetes mellitus; EP: embolismo periférico; FA: fibrilación auricular; IC: insuficiencia cardíaca; TEV: tromboembolismo venoso. 


\subsubsection{Manejo del tratamiento anticoagulante oral en el perioperatorio}

Según el protocolo vigente en nuestro Servicio de Hematología-Hemoterapia en pacientes con FA que precisan la retirada del tratamiento anticoagulante oral previo a un procedimiento invasivo programado, se debe suspender el acenocumarol 3 días antes y debe reiniciarse el día del procedimiento.

Se pauta heparina de bajo peso molecular (HBPM) a dosis profilácticas (enoxaparina $40 \mathrm{mg} /$ día subcutánea) desde 3 días antes de procedimiento hasta 3 días después (de tal manera que se producirá un solapamiento con el TAO en los 3 días posteriores al procedimiento).

Previo a cualquier intervención debe comprobarse la normalidad de la coagulación con la realización de un estudio de coagulación o determinación de INR.

\subsection{DEFINICIÓN DE TERAPIA PUENTE}

Se habla de terapia puente cuando se necesita disminuir o anular el efecto de los fármacos anticoagulantes orales AVK ante situaciones de riesgo de sangrado como intervenciones quirúrgicas o exploraciones que puedan requerir biopsias profundas.

Durante este procedimiento puede aumentar temporalmente el riesgo tromboembólico, por lo que en algunos casos, los fármacos AVK se sustituyen por otros fármacos anticoagulantes preferentemente las heparinas de bajo peso molecular (HBPM).

Para el manejo de estas situaciones, el planteamiento inicial del médico debe ser, antes que nada, decidir si es necesario realmente suspender el TAO y esto va a depender del tipo de intervención al que será sometido el paciente. 
En segundo lugar, una vez indicada la suspensión del tratamiento, debe decidir si es necesario pautar HBPM; esto dependerá del riesgo trombótico de la indicación por la que está anticoagulado el paciente.

Aunque las recomendaciones de las guías clínicas (86) están en general basadas en datos limitados de baja calidad $(87,88)$, existen resultados de ensayos aleatorizados (89) en los que en pacientes con FA, se objetiva una mayor tasa de hemorragias en aquellos pacientes en los que se administra terapia puente frente a los que se interrumpen los AVK sin sustituirlos por otros fármacos, observándose además una similar incidencia de episodios tromboembólicos.

Esto sugiere que en pacientes anticoagulados por FA no valvular de bajo riesgo no se debería administrar terapia puente con HBPM, cuando se suspenda el TAO.

\subsection{HEPARINA DE BAJO PESO MOLECULAR.}

Las heparinas de bajo peso molecular (HBPM) se están utilizando desde la década de los 90; a finales de los años 60 y durante la década de los años 70 se produjeron numerosos hallazgos sobre las propiedades anticoagulantes de estos fármacos (90).

En 1982, se publicaron los primeros datos sobre la eficacia de la HBPM en la prevención de la trombosis venosa profunda en el postoperatorio de cirugía abdominal (91) y, posteriormente en cirugía electiva de cadera (92).

El ímpetu para el desarrollo de heparinas de bajo peso molecular (HBPM) como potenciales antitrombóticos, se objetivó en dos observaciones: el primero fue el hallazgo de que las fracciones de bajo peso molecular obtenidas a partir de heparina estándar (HS) pierden progresivamente su capacidad para prolongar el tiempo parcial de tromboplastina activada (APT a), al tiempo que conservan su capacidad para inhibir el factor $X$ activado (factor $\mathrm{Xa})(93,94)$. 
La segunda observación mostraba que para un efecto antitrombótico equivalente a la heparina no fraccionada, las HBPM producen menos sangrado en los modelos experimentales (95).

Posteriormente, se describió el mecanismo que diferencia el perfil anticoagulante de la HNF y la HBPM y las diferencias en cuanto a su farmacocinética y biodisponibilidad y la variabilidad de su respuesta anticoagulante a dosis fijas.

Así mismo, se desarrollaron varias HBPM que demostraron que son seguras y efectivas en la prevención y el tratamiento del tromboembolismo venoso y con una ventaja añadida sobre la HNF, su uso vía subcutánea (96).

La dosis de la HBPM se ajusta al peso del paciente y se administrada subcutáneamente una o dos veces al día sin monitorización del laboratorio (97).

La mayor experiencia de su uso se consiguió en la profilaxis de trombosis venosa en pacientes de alto riesgo para desarrollar esta complicación y posteriormente, se realizaron estudios en el tratamiento de la trombosis venosa profunda (TVP) con estos fármacos (98).

En los primeros años de su lanzamiento, el uso quedó restringido al ámbito hospitalario pero posteriormente la eficacia y seguridad que demostraron, llevó a su amplia difusión en el ámbito domiciliario con el diseño de estudios sobre el tratamiento ambulatorio de la TVP $(99,100)$.

En los años posteriores, se generalizó su uso como profilaxis extendida de complicaciones trombóticas en pacientes en el domicilio que habían sido sometidos a técnicas quirúrgicas que condicionaban el uso prolongado de HBPM; en estos pacientes, numerosas publicaciones habían alertado de la necesidad de prolongación del tratamiento tras el postoperatorio durante 3 o 4 semanas (101) y además, con un coste razonable (102). 
La profilaxis de complicaciones tromboembólicas se extendió paulatinamente a los pacientes con patologías médicas que condicionaban reposo o inmovilidad prolongados (103) tanto en pacientes hospitalizados como en pacientes inmovilizados en el domicilio (104).

La disponibilidad de las jeringas precargadas y la facilidad de manejo del capuchón que protege la aguja con sistemas de seguridad que retraen la aguja o la ocultan, ha supuesto el uso universal de las HBPM.

Tras años de su uso a nivel domiciliario ya no es el personal sanitario de los Centros de Salud el que habitualmente pone la inyección al paciente sino que es el propio paciente el que se administra el tratamiento tras un pequeño adiestramiento en la técnica correcta de inyección por el personal sanitario.

\subsection{MANEJO DE LA HEMORRAGIA PERIPROCEDIMIENTO}

El manejo de la hemorragia perioperatoria implica múltiples evaluaciones y estrategias para garantizar el apropiado cuidado del paciente por lo que inicialmente es importante identificar a aquellos pacientes con un mayor riesgo de hemorragia perioperatoria.

Así, el esfuerzo de las sociedades científicas ha ido dirigido a proporcionar respuestas clínicas al uso de los anticoagulantes orales y diseñar algoritmos y protocolos sencillos aplicables a la práctica diaria (105).

El manejo periprocedimiento de los pacientes que reciben tratamiento crónico con anticoagulantes orales con los AVK es un problema común y el manejo óptimo de estos pacientes está basado en 4 pilares fundamentales:

- Estratificación del riesgo del paciente en relación con el riesgo de trombosis o hemorragia relacionado con el procedimiento. 
- Las consecuencias clínicas del evento trombótico o hemorrágico.

- La discontinuación o reinicio del TAO en base a las propiedades farmacocinéticas de cada agente.

- Valoración del si el uso periprocedimiento de la terapia puente con heparina de bajo peso molecular tiene ventajas en la prevención del tromboembolismo postoperatorio frente al posible incremento del riesgo hemorrágico.

Este último punto ha generado una gran controversia habiendose pasado desde una posición del uso de terapia puente de manera indiscriminada en todos los pacientes, a definir grupos de riesgo en los cuales la evidencia científica avala no utilizar la terapia puente si el riesgo de tromboembolismo en el periprocedimiento es bajo.

\subsection{USO DE TERAPIA PUENTE}

Los pacientes tratados con anticoagulantes orales antagonistas de la vitamina $\mathrm{K}$ tienen un riesgo incrementado de hemorragia en el peri y post procedimiento.

Si el ratio normalizado internacional (INR) es $\leq 1.5$, la cirugía puede llevarse a cabo de forma segura.

Sin embargo, los pacientes cuyo diagnóstico condiciona un alto riesgo de sufrir un tromboembolismo son aquellos que tienen alguna de estas características:

-FA con $\mathrm{CHA}_{2} \mathrm{DS}_{2}-\mathrm{VASc} \geq 4$

-Prótesis mecánicas, prótesis biológica nuevas, o

-Recambio mitral (en los 3 meses anteriores) o

-Tromboembolismo reciente (en los 3 meses anteriores) o

-Presencia de trombofilia 
En estos casos la discontinuación del AVK es peligrosa y estos pacientes necesitarán un tratamiento puente con heparina de bajo peso molecular que sustituya al anticoagulante oral (106).

\subsection{CONFERENCIAS DE CONSENSO}

El tratamiento anticoagulante oral frecuentemente requiere su interrupción frente a procedimientos invasivos y cirugía.

Si es necesaria o no la terapia puente es un dilema común y la evidencia de la información de la que se dispone para soportar la toma de decisiones es limitada, haciendo que algunas de las guías actuales sean imprecisas y ambiguas.

Por otra parte, las indicaciones para la interrupción de la anticoagulación pueden ser poco claras. Estudios observacionales y recientes estudios aleatorizados han señalado un aumento del porcentaje de hemorragia en el perioperatorio o periprocedimiento sin reducción del tromboembolismo cuando se usa la terapia puente.

Además la sintomatología hemorrágica puede incrementar la mortalidad y morbilidad.

A la luz de estos hallazgos, las preferencias médicas por la terapia puente usada de forma rutinaria, se revela como una práctica demasiado agresiva en un determinado grupo de pacientes.

El estudio aleatorizado "PERIOP2" (A Double Blind Randomized Control Trial of Post-Operative Low Molecular Weight Heparin Bridging Therapy Versus Placebo Bridging Therapy for Patients Who Are at High Risk for Arterial Thromboembolism), ha permitido ayudar en el diseño de una guía del manejo periprocedimiento del TAO para indicaciones como tromboembolismo venoso y válvulas cardíacas mecánicas (107). 
Se han publicado diferentes ensayos aleatorizados en pacientes que reciben AVK y que son sometidos a implantación de marcapasos o desfibrilador en los cuales se usaba la terapia puente en estos procedimientos electivos; el análisis de los mismos ha permitido ponderar el uso de esta práctica y consecuentemente la actualización de las guías de manejo periprocedimiento(108).

Son guías que sufren revisiones y actualizaciones periódicas a medida que van publicándose datos que apoyan las modificaciones de las mismas. Así en el caso de pacientes que requieren implantación de marcapasos o desfibrilador automático implantable (DAI) y están tomando warfarina las guías recomendaban el uso de terapia puente; sin embargo, publicaciones de series de casos sugieren que puede ser seguro la realización de la cirugía sin interrupción del tratamiento anticoagulante oral (109).

La preocupación por el aumento de complicaciones hemorrágicas en el periprocedimiento llevó a la publicación de numerosas guías de actuación sobre el manejo perioperatorio de los pacientes con FA en tratamiento anticoagulante oral $(110,111)$.

También en el caso de que los pacientes requirieran cirugía dental, las sociedades de odontología emitieron unas guías con el objetivo de procurar a los odontólogos y otros profesionales de la salud, una orientación clara en el manejo de los pacientes en tratamiento con anticoagulantes orales que debían ser sometidos a este tipo de cirugía (112).

El estudio "The Outcomes Registry for Better Informed Treatment of Atrial Fibrillation (ORBIT-AF)", es un registro prospectivo y observacional de pacientes con FA en los cuales se han producido interrupciones temporales del TAO debido a diferentes procedimientos y en el que se recoge el tipo de terapia puente.

Los resultados incluyen un análisis multivariante de las complicaciones tromboembólicas como infarto de miocardio, ictus o embolismo sistémico, 
complicaciones hemorrágicas, causas de hospitalización y muerte en los 30 días posteriores al procedimiento. En 7.372 pacientes tratados con TAO, se recogieron 2.803 eventos de interrupción de la anticoagulación en 2.200 pacientes (30 \%) con un seguimiento de 2 años; se utilizó la terapia puente en el $24 \%$ ( $n=665)$, fundamentalmente heparina de bajo peso molecular $(73 \%, n=487)$ y en menor proporción la heparina no fraccionada $(15 \%, n=97)$.

Se observó que los pacientes que recibieron terapia puente presentaban en sus antecedentes mayor proporción de eventos cerebrovasculares y $(22 \%$ versus $15 \%$; $\mathrm{P}=0.0003$ ) y prótesis valvular mecánica (9.6\% versus $2.4 \%$; $\mathrm{P}<0.0001$ ); sin embargo, no se detectaron diferencias en cuando a la escala $\mathrm{CHA}_{2} \mathrm{DS}_{2}$-VASc (scores $\geq 2$ in $94 \%$ versus $95 \% ; \mathrm{P}=0.5)$.

Los eventos hemorrágicos fueron más comunes en los pacientes sometidos a la terapia puente que en los que no la recibieron (5.0\% versus $1.3 \%$; odds ratio ajustada, 3.84; $P<0.0001)$.

Con respecto a la incidencia de infarto de miocardio, ictus o embolismo sistémico, hemorragia mayor, hospitalización o muerte tras los 30 días del procedimiento, se observó que fue también significativamente mayor en pacientes que recibieron la terapia puente (13\% versus 6.3\%; odds ratio ajustada, 1.94; $\mathrm{P}=0.0001)$.

En este estudio de objetivó que la terapia puente era usada en un $25 \%$ de las interrupciones del TAO y estaba asociada con un mayor riesgo de hemorragia y eventos adversos. Por tanto, estos datos no apoyarían el uso rutinario de la terapia puente (113).

Estos datos ponían de manifiesto la dudosa necesidad de la terapia puente en pacientes con FA que precisan la interrupción del tratamiento anticoagulante con AVK ante un procedimiento electivo invasivo; por ello se planteó la hipótesis de que 
abstenerse de la terapia puente podría "no ser inferior" al uso de terapia puente con heparina de bajo peso molecular para la prevención del tromboembolismo perioperatorio y abstenerse podría "ser superior" al uso de terapia puente con respecto a la complicación de hemorragia mayor.

Para aclarar este tema, Douketis et al diseñaron el estudio BRIDGE, aleatorizado, doble ciego, controlado con placebo en el que después de la interrupción perioperatoria de la terapia con warfarina, los pacientes fueron asignados al azar para recibir terapia puente con heparina de bajo peso molecular o placebo; los autores pretendían definir la necesidad de la terapia puente en pacientes con FA sometidos a procedimientos invasivos. Los objetivos del estudio incluían valorar la aparición de tromboembolismo arterial y sangrado mayor en los 30 días posteriores al procedimiento.

El reclutamiento incluyó 1.884 pacientes, 950 asignados a recibir placebo y 934 a recibir terapia puente.

Se objetivó una incidencia de tromboembolismo del 0,4\% en el primer grupo y del 0,3\% en el que recibió terapia puente (diferencia de riesgo, 0.1 puntos; 95\% intervalo de confianza $[\mathrm{Cl}],-0.6$ a $0.8 ; \mathrm{P}=0.01$ para no inferioridad).

La incidencia de hemorragia mayor fue $1.3 \%$ en el grupo sin terapia puente y $3.2 \%$ en el grupo de terapia puente (riesgo relativo, $0.41 ; 95 \% \mathrm{Cl}, 0.20$ hasta 0.78 ; $\mathrm{P}=0.005$ por superioridad).

Con estos datos, los autores concluyeron que el grupo de pacientes que no recibió terapia puente no solo no presentó mayores complicaciones tromboembólicas sino que además, tuvo un menor riesgo de sangrado (89).

Finalmente, en las recomendaciones del manejo perioperatorio del tratamiento anticoagulante oral publicadas por el American College of Chest Physicians Evidence- 
Based Clinical Practice Guidelines en su 9th edición, Douketis et al, consideran que los pacientes con FA y CHADS2 escore 0 a 2 son de bajo riesgo para sufrir un tromboembolismo cuando se retira la anticoagulación oral puesto que tendrían solo un $5 \%$ de riesgo anual de presentar complicaciones tromboembólicas tras la suspensión.

Por ello, en estos pacientes no recomiendan el uso de terapia puente (1). 
3. HIPÓTESIS 


\section{HIPÓTESIS}

En este proyecto se plantean las siguientes hipótesis de trabajo:

\section{Hipótesis principal:}

El uso de terapia puente con heparina de bajo peso molecular en pacientes con FA de bajo riesgo que precisan la retirada del tratamiento con AVK ante un procedimiento invasivo programado es innecesaria puesto que el riesgo trombótico tras el procedimiento es mínimo.

\section{Hipótesis secundarias:}

La administración de heparina de bajo peso molecular como terapia puente en pacientes con FA de bajo riesgo que precisan la retirada del tratamiento con AVK ante un procedimiento invasivo programado aumenta el riesgo de complicaciones hemorrágicas.

La administración de heparina de bajo peso molecular como terapia puente en pacientes con FA de bajo riesgo que precisan la retirada del tratamiento con AVK ante un procedimiento invasivo programado aumenta el coste del procedimiento. 
4. OBJETIVOS 


\section{OBJETIVOS}

El objetivo principal de la presente tesis doctoral es:

-Determinar la utilidad de la terapia puente en pacientes con FA de bajo riesgo en un procedimiento invasivo programado.

El objetivo secundario de la tesis es:

-Determinar las complicaciones hemorrágicas generadas por el uso de la terapia puente.

-Determinar el coste generado por el uso de la terapia puente. 


\section{MATERIAL Y MÉTODOS}




\section{MATERIAL Y MÉTODOS}

\subsection{DISEÑO DEL ESTUDIO}

Considerando la hipótesis y los objetivos previamente marcados, se ha diseñado un estudio de tipo retrospectivo y observacional.

\subsection{DESCRIPCIÓN DE LA POBLACIÓN}

\subsubsection{Población de estudio}

Pacientes diagnosticados de Fibrilación auricular de bajo riesgo en tratamiento anticoagulante oral con acenocumarol que precisaron la retirada de dicho tratamiento previoa la realización de un procedimiento invasivo programado.

Dentro del grupo de bajo riesgo se incluían los pacientes con FA y $\mathrm{CHADS}_{2}$ score 0 a 2 (sin ictus previo ni accidente isquémico transitorio).

\subsubsection{1. Ámbito del estudio}

El periodo de estudio comprende a los pacientes que precisaron la retirada de la anticoagulación oral con acenocumarol a los largo de un año y cuyo control anticoagulante se realizó por los médicos de la Unidad de Anticoagulación oral del Servicio de Hematología-Hemoterapia del Hospital Universitario de Burgos.

\subsubsection{Criterios de inclusión}

Pacientes de ambos sexos sin límite de edad con diagnóstico de Fibrilación auricular de bajo riesgo en tratamiento anticoagulante oral con acenocumarol y que 
precisaron la retirada del tratamiento anticoagulante oral de cara a la realización de un procedimiento invasivo programado.

\subsubsection{Criterios de exclusión}

Pacientes en tratamiento anticoagulante oral con acenocumarol por un diagnóstico diferente al de Fibrilación auricular de bajo riesgo.

\subsubsection{Método de recogida de información}

Los pacientes del estudio se encuentran incluidos en la base de datos del programa de tratamiento anticoagulante oral $\mathrm{TAONET}^{\circledR}$, que es el utilizado para pautar la dosis del tratamiento anticoagulante oral en la Unidad de Anticoagulación oral del Servicio de Hematología-Hemoterapia del Hospital Universitario de Burgos.

Se seleccionaron aquellos que precisaron la retirada del tratamiento anticoagulante oral ante un procedimiento invasivo programado.

En la Unidad de Anticoagulación oral se ha descentralizado la punción capilar y la información del paciente, de tal manera que desde el Centro de Salud el personal de enfermería incorpora el INR y los comentarios de relevancia clínica para el tratamiento anticoagulante oral, en la ficha electrónica del paciente.

El programa informático TAONET ${ }^{\circledR}$ permite añadir en el apartado de “Comentario" incidencias tales como cambios en el tratamiento habitual, procesos intercurrentes así como la necesidad de retirada de este fármaco de cara a una intervención quirúrgica u otro procedimiento invasivo.

Posteriormente es el Médico Hematólogo el que decide la dosis y próxima cita del paciente; de la misma manera la retirada del acenocumarol y la sustitución por otro 
anticoagulante también es responsabilidad del especialista: éste pautará la terapia puente ante cualquier procedimiento invasivo programado si lo considera recomendado.

Según el diagnóstico y el procedimiento invasivo, el período de suspensión del anticoagulante es variable. Los pacientes se clasifican en bajo y alto riesgo trombótico según el diagnóstico por el que se indica el tratamiento anticoagulante e implican una intensidad de anticoagulación diferente, siendo mayor en el caso de un diagnóstico de alto riesgo.

Así mismo, el riesgo trombótico del diagnóstico condiciona la dosis de heparina de bajo peso molecular que se utilizará en la terapia puente si ésta es necesaria.

Posteriormente, se completó la búsqueda de complicaciones trombóticas con el análisis de la historia clínica del paciente. Actualmente, en el Hospital Universitario de Burgos está vigente la historia clínica electrónica con el aplicativo Jimena ${ }^{\circledR}$.

Se hizo una revisión de la misma enfocada a la recogida de complicaciones trombóticas o hemorrágicas en los 30 días posteriores a la interrupción de la anticoagulación oral.

Tanto el programa informático de tratamiento anticoagulante oral, TAONET $^{\circledR}$ como $^{-1}$ el programa informático de historia clínica Jimena ${ }^{\circledR}$, utilizan el mismo número de historia clínica para codificar la información de los pacientes, lo cual simplifica el proceso de búsqueda de información.

Se hizo una recogida de datos epidemiológicos, de la indicación del tratamiento anticogulante oral, del procedimiento quirúrgico o invasivo y de la pauta de retirada de la anticoagulación. 
Toda la información referente al estudio de incluyó en una base de datos en formato Excel que permitió su exportación posterior al programa estadístico SPSS con el que se realizó el análisis de los resultados.

\subsubsection{Información recogida}

Se recogieron los siguientes datos:

- Edad

- Sexo

- Diagnóstico por el que precisaba el tratamiento anticoagulante oral

- Procedimiento invasivo al que iba a ser sometido

- Uso de terapia puente

- Complicaciones hemorrágicas

- Complicaciones trombóticas

- Valoración en el Servicio de Urgencias por la complicación presentada

- Necesidad de ingreso hospitalario debido a la complicación

- Necesidad de transfusión por la aparición de una complicación hemorrágica

- Días de ingreso hospitalario generados por la complicación hemorrágica o trombótica

- Muerte ocasionada por la complicación

- Coste del uso de terapia puente

- Coste de la atención en el Servicio de Urgencias

- Coste del ingreso hospitalario.

\subsection{MÉTODO DE RECOGIDA Y TRATAMIENTO DE LOS DATOS}

La recogida retrospectiva se realizó mediante la revisión de la historia electrónica de tratamiento anticoagulante oral en el programa TAONET $^{\circledR}$. 
La información sobre las complicaciones presentadas se obtuvo de la revisión de historias clínicas en formato electrónico en el programa Jimena ${ }^{\circledR}$.

\subsubsection{Entrada y gestión informática de los datos}

Toda la información referente al estudio de incluyó en una base de datos en formato Microsoft Office Excel 2010 que permitió su exportación posterior al programa estadístico IBM SPSS 19 con el que se realizó el análisis de los resultados (con un intervalo de confianza del 95\%).

Se realizó un análisis descriptivo de la muestra aportando medias y desviación estándar (para el caso de la edad) y frecuencia absolutas (frecuencias relativas) para el resto de variables.

\subsection{VARIABLES}

En este apartado se detallan los criterios utilizados para clasificar la patología de los pacientes.

Se recogieron datos correspondientes a la filiación del paciente:

- Edad

- Sexo

- Diagnóstico por el que precisaba el tratamiento anticoagulante oral

- Procedimiento invasivo al que iba a ser sometido

- Uso de terapia puente

- Complicaciones hemorrágicas: diferenciando:

-Hemorragia mayor: La definición de sangrado mayor incluye: hemorragia intracraneal, sangrado retroperitoneal, descenso del hematocrito mayor o igual al 
$12 \%$, transfusión de hematíes cuando el hematocrito es mayor o igual al $28 \%$ o transfusión cuando el hematocrito es menor al $28 \%$ y el sangrado es evidente.

-Hemorragia menor: incluye hemorragias a distintos niveles que no comprometen la vida del paciente ni requieren transfusión (Epistaxis, equimosis, hematuria).

- Complicaciones trombóticas: incluyendo

- Cualquier patología trombótica producida en el periprocedimiento tanto trombosis venosa (tromboembolismo pulmonar, trombosis venosa profunda) como arterial (isquemia mesentérica o cerebrovascular).

- Valoración en el Servicio de Urgencias por la complicación presentada

- Necesidad de ingreso hospitalario debido a la complicación

- Necesidad de transfusión por la aparición de una complicación hemorrágica

- Días de ingreso hospitalario generados por la complicación hemorrágica o trombótica

- Muerte ocasionada por la complicación.

\subsubsection{Variables a estudio}

A continuación se enumeran las variables independientes que se recogieron en el estudio. Las variables cualitativas se clasificaron en distintas categorías y se detallan las unidades en las que se recogieron las variables cuantitativas.

Datos epidemiológicos de los pacientes:

- Código de identificación

- Sexo.
○ Varón
○ Mujer

- Fecha de nacimiento. 
Diagnóstico por el que precisaba el tratamiento anticoagulante oral

Procedimiento invasivo al que iba a ser sometido:

- Intervención quirúrgica menor

- Colonoscopia:

Realización de polipectomía

Realización de biopsia

Estudio sin biopsia ni polipectomía

- Gastroscopia:

Realización de biopsia

Estudio sin biopsia

- Biopsia de próstata

- Biopsia de mama

- Infiltración

- Otro procedimiento invasivo

Uso de terapia puente

Complicaciones hemorrágicas:

- Hemorragia mayor:

○ $\mathrm{Si}$

○ No

- Hemorragia menor

○ Si

○ No

Complicaciones trombóticas
○ Si
○ No

Valoración en el Servicio de Urgencias por la complicación presentada.
○ $\mathrm{Si}$
○ No 
Necesidad de ingreso hospitalario debido a la complicación
$\circ \mathrm{Si}$
○ No

Necesidad de transfusión por la aparición de una complicación hemorrágica
○ $\mathrm{Si}$
○ No

Días de ingreso hospitalario generados por la complicación hemorrágica o trombótica Resolución total o con secuelas

Muerte ocasionada por la complicación.
$\circ \mathrm{Si}$
○ No

\subsubsection{Variables analíticas}

En las complicaciones hemorrágicas se recogieron las siguientes variables:

- Hemograma: cifra de hemoglobina

- Bioquímica: creatinina, GPT

- Número de unidades transfundidas

\subsubsection{Variables resultado}

- Complicaciones hemorrágicas

- Complicaciones trombóticas

- Uso del Servicio de Urgencias

- Ingreso hospitalario

- Estancia hospitalaria (días)

- Resolución de la complicación

- Éxitus. 


\subsection{ANÁLISIS DEL COSTE}

\subsubsection{Análisis del coste de la terapia puente}

Se realizó una valoración del coste del uso de la terapia puente con heparina de bajo peso molecular, teniendo en cuenta la pauta habitual seguida por el Servicio de Hematología-Hemoterapia del Hospital Universitario de Burgos.

Según dicha pauta se utiliza una inyección diaria de HBPM a dosis profiláctica (enoxaparina $40 \mathrm{mg}$ ) los 3 días previos al procedimiento y los 3 días posteriores al mismo, de tal manera que cada paciente recibió 6 inyecciones con jeringa precargada.

Se solicitó información al Servicio de Farmacia para conocer el precio de la unidad de HBPM que fue de 4,5 euros.

\subsubsection{Análisis del coste de las complicaciones}

Para valorar este coste se solicitó al Servicio de Gestión del Hospital Universitario de Burgos el coste de asistencia en el Servicio de Urgencias y el coste por ingreso hospitalario.

El coste de la atención en el servicio de Urgencias fue 118,68 por paciente.

El coste del ingreso hospitalario por día fue de 623,71 euros.

\subsection{ASPECTOS ÉTICOS}

Este trabajo se llevó a cabo de conformidad con todas las leyes y normativas aplicables, de acuerdo a los principios éticos internacionales, fundamentalmente la 
Declaración de Helsinki (Fortaleza, Brasil, 2013) y las Normas de Buena Práctica Clínica Epidemiológicas de la ICH (International Conference of Harmonization).

Todos los pacientes del estudio fueron identificados mediante un código de identificación. Los datos fueron tratados con absoluta confidencialidad, según la Ley Orgánica 15/1999, de 13 de diciembre, de protección de datos de carácter personal.

El estudio se realizó según lo establecido en este protocolo, una vez evaluado y aprobado por el Comité Ético de Investigación Clínica (CEIC) del CEIC Área de Salud de Burgos y Soria.

Al preservar la identidad de los participantes el Comité Ético permitió la realización del estudio sin consentimiento informado.

\subsection{FINANCIACIÓN}

Este estudio ha recibido una beca para su realización de la Fundación Burgos para la Investigación de la Salud (FBIS 2015).

\subsection{BUSQUEDA BIBLIOGRÁFICA}

La búsqueda bibliográfica principal ha sido realizada en la base de datos MEDLINE a través de PubMed (que puede consultarse en: http://www.ncbi.nlm.nih.gov/pubmed/), recurso de acceso libre mantenido y desarrollado por el Centro Nacional de Información Biotecnológica (National Center for Biotechnology Information, NCBI), de la Biblioteca Nacional de Medicina (National Library of Medicine, NLM) de los Estados Unidos, localizada en los Institutos Nacionales de Salud (National Institutes of Health, NIH). La búsqueda inicial se planteó utilizando 
términos libres y la definitiva a través de términos controlados tomados del tesauro de MEDLINE, la base de datos MeSH.

Además, se han realizado búsquedas secundarias en las bases de datos COCHRANE y EMBASE, utilizando las mismas estrategias, adaptadas a las características específicas de dichas bases.

La revisión bibliográfica ha sido realizada en dos tiempos, el primero antes del diseño del estudio, para lograr una idea del estado actual del tema, y el segundo con posterioridad al mismo, con el fin de priorizar los datos obtenidos y establecer modelos comparativos.

La revisión ha sido completada con trabajos localizados en las fuentes anteriores, más la consulta de libros de texto actualizados de referencia en el tema tratado, así como de guías clínicas.

Todas las referencias han sido incluidas en una base de datos del programa de gestión de referencias bibliográficas Mendeley. Para su redacción, se utilizó el denominado estilo Vancouver, recomendado por el Comité Internacional de Editores de Revistas Médicas (International Committee of Medical Journal Editors, ICMJE), cuyos requerimientos de uniformidad para manuscritos, revisados en diciembre de 2018, pueden encontrarse en http://www.icmje.org/icmje-recommendations.pdf (acceso 01/12/2018), con ejemplos de las referencias más típicamente utilizadas en artículos científicos disponibles en http://www.nlm.nih.gov/bsd/uniform requirements.html (acceso 01/12/2018). 
6. RESULTADOS 


\subsection{ANÁLISIS DEL GRUPO DE ESTUDIO}

\subsubsection{Análisis del grupo completo}

En el periodo a estudio, durante 2013, se retiró el tratamiento anticoagulante oral a un total de 220 pacientes con una edad media de 73,9 años de los cuales 92 (42\%) eran mujeres y 128 hombres (58\%) .

Los diagnósticos por los que los pacientes recibían tratamiento anticoagulante fueron los siguientes:

- Fibrilación auricular: 161 pacientes

- Tromboembolismo pulmonar y/o trombosis venosa profunda: 19 pacientes

- Accidentes cerebrovascular: 3

- Prótesis mitral: 17

- Prótesis aórtica: 12

- Otros diagnósticos: 8

Los procedimientos que motivaron la retirada del tratamiento anticoagulante oral se detallan a continuación:

- Intervención quirúrgica menor: 70

- Colonoscopia: 94

○ Realización de polipectomía: 54

○ Sin polipectomía: 5

○ Biopsia: 15

O Solo estudio: 20

- Gastroscopia: 15
○ Biopsia: 9
○ No biopsia: 6

- Biopsia de próstata: 7 
- Biopsia de mama: 6

- Infiltración: 6

- Otro procedimiento: 22.

\subsubsection{Grupo con diagnóstico de FA y bajo riesgo.}

En el periodo a estudio, durante 2013 , se retiró el tratamiento anticoagulante oral a un total de 161 pacientes con diagnóstico de FA y $\mathrm{CHADS}_{2}$ score 0 a 2, con una edad media de 76,11 años $( \pm 8,45)$ de los cuales 64 (40 \%) eran mujeres con una edad media de 77,03 años $( \pm 8,09)$ y 97 hombres (60\%) con una edad media de 75,51 años $( \pm 8,66)$.

Tabla 4. Características de edad y sexo de los pacientes

\begin{tabular}{lccc}
\hline Variable & Mujeres $(\mathbf{n} / \%)$ & Hombres $(\mathrm{n} / \%)$ & Total $(\mathrm{n} / \%)$ \\
\hline Sexo & $64(40)$ & $97(60)$ & $161(100)$ \\
& & & \\
Edad & $77.03( \pm 8,09)$ & $75.51( \pm 8,66)$ & $76,11( \pm 8,45)$ \\
\hline
\end{tabular}

A continuación se detallan los procedimientos que motivaron la retirada del tratamiento anticoagulante oral:

- Intervención quirúrgica menor: 58 (36 \%)

- Colonoscopia: 61 (38\%)

○ Realización de polipectomía: 36

○ $\quad$ Sin polipectomía: 2 
- Biopsia: 10

- Solo estudio: 13

- Gastroscopia: 11 (7\%)

○ Biopsia: 8

○ No biopsia: 3

- Biopsia de próstata: 4 (2,5\%)

- Biopsia de mama: 4 (2,5\%)

- Infiltración: 5 (3 \%)

- Otro procedimiento: 18 (11\%)

En la siguiente tabla se exponen los procedimientos invasivos programados:

Tabla 5. Procedimientos invasivos

\begin{tabular}{|c|c|c|c|}
\hline Variable & Mujeres (n/\%) & Hombres (n/\%) & Total (n/\%) \\
\hline Sexo & $64(40)$ & $97(60)$ & $161(100)$ \\
\hline $\begin{array}{l}\text { Procedimiento } \\
\text { invasivo: }\end{array}$ & & & \\
\hline $\begin{array}{l}\text { Intervención } \\
\text { quirúrgica menor }\end{array}$ & $23(39.6)$ & $35(60.4)$ & $58(36)$ \\
\hline Colonoscopia & $24(39.3)$ & $37(60.7)$ & $61(38)$ \\
\hline Gastroscopia: & $6(54.5)$ & $5(45.5)$ & $11(7)$ \\
\hline $\begin{array}{r}\text { Biopsia de } \\
\text { próstata }\end{array}$ & & $4(2.5)$ & $4(2.5)$ \\
\hline Biopsia de mama & $4(2.5)$ & & $4(2.5)$ \\
\hline Infiltración* & 0 & $5(3)$ & $5(3)$ \\
\hline $\begin{array}{r}\text { Otro } \\
\text { procedimiento** }\end{array}$ & $7(38.8)$ & $11(61.2)$ & $18(11)$ \\
\hline
\end{tabular}


*Infiltración en rodilla, por neuropatía de nervio safeno izquierdo, en esfínter anal interno con toxina botulínica, en tobillo, en nervio femorocutáneo izquierdo.

**Otro tipo de procedimiento: biopsia de glándula salival, implante intraocular de dispositivo liberador de corticoide, cistoscopia (5 casos), biopsia de cavum, biopsia pulmonar, punción aspiración de nódulo tiroideo (3 casos), broncoscopia, punción lumbar, biopsia endometrial, punción aspiración de masa pancreática, punción aspiración de masa renal y biopsia de ganglio centinela).

\subsection{COMPLICACIONES HEMORRÁGICAS O TROMBÓTICAS}

No se constató ninguna complicación en 155 (96\%) pacientes y hubo 6 (4\%) pacientes que presentaron complicaciones: 5 hemorrágicas y 1 tromboembólica.

\subsubsection{Análisis de las complicaciones hemorrágicas}

Con respecto a las complicaciones hemorrágicas destacar que se objetivó una hemorragia mayor y 4 hemorragias menores.

A continuación se exponen con más detalle:

1. mujer presentó una hemorragia mayor a los 12 días de la colonoscopia y polipectomía por lo que requirió ingreso durante 6 días y transfusión de 1 concentrado de hematíes (Hb pretransfusional: $91 \mathrm{gr} / \mathrm{L}$ ).

2. mujer tuvo una hemorragia menor tras una intervención de cirugía menor del túnel carpiano a los 5 días del procedimiento; la paciente fue valorada en el Servicio de Urgencias no requiriendo tratamiento específico ni ingreso hospitalario.

3. varón sometido a una intervención quirúrgica menor por una lesión en piel con sospecha de Carcinoma Basocelular; a las 24 horas acudió al Servicio de Urgencias por hematoma en la zona de la cirugía y tras valoración, fue dado de alta. 
4. varón al que se le realizó una colonoscopia a través de la colostomía e ingresó 26 días después por hemorragia por la colostomía; requirió 3 días de ingreso pero no precisó transfusión aunque la cifra de hemoglobina descendió 20 gr/L (Hb previa al procedimiento de $143 \mathrm{gr} / \mathrm{L}$ y Hb $123 \mathrm{gr} / \mathrm{L}$ tras el procedimiento).

5. varón sometido a colonoscopia y polipectomía; ingresó a las 48 horas con hemorragia menor (Hb previa $101 \mathrm{gr} / \mathrm{L}$ y posterior $96 \mathrm{gr} / \mathrm{L}$ ); estuvo ingresado 2 días.

\subsubsection{Análisis de las complicaciones trombóticas}

Un paciente presentó una complicación trombótica como se detalla a continuación:

1. varón al que se le realizó una biopsia de próstata e ingresó el mismo día del procedimiento por un Ictus subagudo cerebeloso izquierdo; requirió 6 días de ingreso y al alta presentaba una recuperación total.

En la siguiente tabla se exponen las características de las complicaciones.

Tabla 6. Características de las complicaciones hemorrágicas y trombóticas

\begin{tabular}{lccc}
\hline Variable & Mujeres (n/\%) & Hombres (n/\%) & Total (n/\%) \\
\hline Sexo & $64(40)$ & $97(60)$ & $161(100)$ \\
No complicaciones & $62(38.5)$ & $93(58.5)$ & $156(96.9)$ \\
Complicaciones: & 2 & 4 & $6(3.7)$ \\
Hemorragia mayor & $1(0.6)$ & 0 & $1(0.6)$ \\
Hemorragia menor & $1(0.6)$ & $3(1.8)$ & $4(2.4)$ \\
Complicaciones & 0 & $1(0.6)$ & $1(0.6)$ \\
\hline trombóticas & & & \\
\hline
\end{tabular}




\subsection{ANÁLISIS DE EFECTOS SECUNDARIOS POR EL USO DE HBPM}

No hemos detectado efectos secundarios derivados del uso de la HBPM a dosis profilácticas. Los pacientes no informaron de la aparición de hematomas en el lugar de la punción ni del dolor ocasionado por la inyección.

\subsection{ANÁLISIS DEL COSTE}

\subsubsection{Análisis del coste de la terapia puente}

Se realizó una valoración del coste del uso de la terapia puente con heparina de bajo peso molecular, teniendo en cuenta la pauta habitual seguida por el Servicio de Hematología del HUBU.

Según dicha pauta se utiliza una inyección de HBPM a dosis profiláctica (enoxaparina $40 \mathrm{mg}$ ) los 3 días previos al procedimiento y los 3 días posteriores al mismo, de tal manera que cada paciente utilizó 6 inyecciones con jeringa precargada.

Se solicitó información al Servicio de Farmacia para conocer el precio de la unidad de HBPM que fue de 4,5 euros.

Se utilizaron 966 dosis de enoxaparina en los 161 pacientes con un coste por unidad de 4,5 euros lo que supuso un gasto total de 4.347 euros.

\subsubsection{Análisis del coste de las complicaciones}

Para valorar este coste se solicitó al Servicio de Gestión del Hospital Universitario de Burgos el coste de asistencia en el Servicio de Urgencias y el coste por ingreso hospitalario. 
El coste de la atención en el Servicio de Urgencias fue 118,68 por paciente, puesto que 2 pacientes fueron atendidos en este servicio, el coste total es de 237,36 euros.

El coste del ingreso hospitalario por día fue de 623,71 euros; las complicaciones hemorrágicas que presentaron los pacientes supusieron 11 días de ingreso, generando un coste total de $6.860,81$ euros.

La suma total del coste generado por la aplicación incorrecta de las recomendaciones fue de $11.445,17$ euros. (Tabla 7 )

Tabla 7. Coste de la terapia puente y las complicaciones

\begin{tabular}{|c|c|c|c|}
\hline & & Euros & Total (euros) \\
\hline $\begin{array}{l}\text { Terapia puente } \\
\text { (HBPM) }\end{array}$ & $\begin{array}{l}966 \text { (6 dosis/161 } \\
\text { pacientes) }\end{array}$ & 4,5/unidad & 4.347 \\
\hline $\begin{array}{l}\text { Servicio de } \\
\text { Urgencias }\end{array}$ & 2 pacientes & $118,68 /$ paciente & 237,36 \\
\hline Hospitalización & $\begin{array}{l}3 \text { pacientes/11 } \\
\text { días }\end{array}$ & 623,71/día & $6.860,81$ \\
\hline Coste total & & & $11.445,17$ \\
\hline
\end{tabular}


7. DISCUSIÓN 


\section{DISCUSIÓN}

La información disponible sobre el manejo periquirúrgico de los anticoagulantes orales es limitada y proviene, fundamentalmente, de estudios observacionales y de recomendaciones de diversas sociedades científicas.

Además, las diferentes situaciones que se pueden plantear durante la actividad quirúrgica inducen a que exista una gran variabilidad en la atención a los pacientes anticoagulados durante la precirugía.

De este modo podemos constatar la enorme disparidad de criterios que existen en el manejo de estos fármacos ante la retirada y sustitución o no por otro anticoagulante vía subcutánea, fundamentalmente HBPM (108).

En las recomendaciones del American College of Chest Physicians (ACCP 2012) se evaluaba el riesgo trombótico de los pacientes sometidos a tratamiento anticoagulante oral cuando este es retirado ante un procedimiento invasivo y se proponían 3 grupos de riesgo en cuanto a la posibilidad de presentar un tromboembolismo tras la retirada de la anticoagulación (1).

Dentro del grupo de bajo riesgo se incluían los pacientes con FA y $\mathrm{CHADS}_{2}$ score 0 a 2 (sin ictus previo ni accidente isquémico transitorio) y pacientes con enfermedad tromboembólica venosa de más de 12 meses y sin otros factores de riesgo.

Sin embargo, la información disponible sobre el manejo periquirúrgico y periprocedimiento invasivo de los anticoagulantes orales AVK, es escasa.

Para aclarar este tema, Douketis et al diseñaron el estudio BRIDGE, aleatorizado, doble ciego, controlado con placebo en el que después de la interrupción perioperatoria de la terapia con warfarina, los pacientes fueron asignados al azar para 
recibir terapia puente con heparina de bajo peso molecular o placebo; los autores pretendían definir la necesidad de la terapia puente en pacientes con FA sometidos a procedimientos invasivos. Los objetivos del estudio incluían valorar la aparición de tromboembolismo arterial y sangrado mayor en los 30 días posteriores al procedimiento.

El reclutamiento incluyó 1.884 pacientes, 950 asignados a recibir placebo y 934 a recibir terapia puente.

Se objetivó una incidencia de tromboembolismo del 0,4\% en el primer grupo y del 0,3\% en el que recibió terapia puente (diferencia de riesgo, 0.1; $95 \%$ intervalo de confianza $[\mathrm{Cl}],-0.6$ a $0.8 ; \mathrm{P}=0.01$ para no inferioridad).

La incidencia de hemorragia mayor fue $1.3 \%$ en el grupo sin terapia puente y $3.2 \%$ en el grupo de terapia puente (riesgo relativo, $0.41 ; 95 \% \mathrm{Cl}, 0.20$ a $0.78 ; \mathrm{P}=0.005$ para superioridad).

Con estos datos, los autores concluyeron que el grupo de pacientes que no recibió terapia puente no solo no presentó mayores complicaciones tromboembólicas sino que además, tuvo un menor riesgo de sangrado (89).

En nuestro análisis utilizando la terapia puente en todos los casos, observamos que la incidencia de tromboembolismo y de hemorragia mayor fue del 0,62 \% y la incidencia de hemorragia menor fue del $2,4 \%$.

En la misma línea que en el estudio BRIDGE, en el estudio retrospectivo de Clark et al, se analizaron un total de 1.812 procedimientos invasivos en pacientes en tratamiento anticoagulante oral por haber presentado una trombosis venosa; en este estudio objetivaron una tasa de hemorragia clínicamente significativa en los 30 días posteriores al procedimiento invasivo del $2.7 \%$ en el grupo de terapia puente frente al $0.2 \%$ en el grupo sin terapia puente y no se encontraron diferencias significativas en el 
número de recurrencias de enfermedad tromboembólica entre ambos grupos. Por tanto, se concluye que la terapia puente se asocia con un incremento del riesgo hemorrágico (114).

La preocupación por las complicaciones derivadas del mal uso de la terapia puente ha llevado a numerosas sociedades científicas a cuestionar esta práctica (115).

Incidiendo en este punto, la Sociedad Hematológica Canadiense sugiere que no se ofrezca la terapia puente a menos que el riesgo trombótico exceda el riesgo de sangrado puesto que la mayoría de los pacientes con riesgo moderado no se van a beneficiar de su uso (116).

En el grupo de los pacientes de bajo riesgo trombótico en los que tras la suspensión del tratamiento anticoagulante oral sufren un episodio trombótico debe hacerse un análisis exhaustivo de los factores de riesgo que hayan podido abocar a esta complicación. Tal vez en un futuro deban diferenciarse los pacientes en grupos de riesgo menos amplios que los actualmente vigentes y deberían entrar en consideración otro tipo de factores de riesgo en estos momentos desconocidos o a los que no se ha dado la suficiente importancia.

En el análisis efectuado por Ríos et al, en pacientes con FA y tratamiento con AVK, el uso de la terapia de puente como parte del manejo clínico durante un procedimiento invasivo se asoció de forma independiente con un mayor riesgo de cualquier evento peri-procedimiento (117).

Un novedoso estudio es el realizado por van der Pol $S$ et al. en el que desarrollaron un modelo para ayudar en la toma de decisiones clínicas comparando el uso de la terapia puente perioperatoria con no pautar esta en pacientes con FA y estando en tratamiento anticoagulante oral con acenocumarol y otros AVK. Observaron que en los pacientes en tratamiento con acenocumarol la terapia puente parece beneficiosa a partir de una puntuación de $\mathrm{CHA}_{2} \mathrm{DS}_{2}$-VASc de 7 (118). 
En nuestro país, los datos muestran que el uso de warfarina es escaso (5,2\%); sin embargo, no se han encontrado diferencias en cuanto a la metodología de control de la anticoagulación oral con respecto al acenocumarol, respaldando esta información la extrapolación que habitualmente se hace de los resultados de los estudios efectuados con warfarina en FA no valvular a los pacientes en tratamiento con acenocumarol (119).

La descentralización del control del tratamiento anticoagulante oral con AVK supuso la asunción de mayores competencias por parte de los médicos de Atención Primaria habiendo demostrado en numerosas publicaciones la calidad en el control de este tipo de tratamiento $(120,121)$.

Además, el coste del control del tratamiento anticoagulante oral en Atención Primaria es menor que en Atención especializada (122) y por ello la implicación de los Médicos de Familia en la implantación de las guías de tratamiento anticoagulante oral es fundamental y supondrá un ahorro en el coste del control de estos pacientes.

En nuestro medio el programa informático permite emitir un informe con las recomendaciones sobre la retirada del anticoagulante y por tanto, no se precisa el ingreso de los pacientes para la realización de la terapia puente; esta práctica no es coste-efectiva ya que los cambios en el tratamiento anticoagulante puede realizarse a nivel ambulatorio como han demostrado Pappas et al (123).

El seguimiento de las guías no solo disminuye las complicaciones o los efectos adversos de un fármaco o procedimiento si no que suponen un importante ahorro (124).

En nuestro estudio el análisis del coste por el uso indebido de la terapia puente con heparina de bajo peso molecular y de la atención sanitaria debida a las complicaciones hemorrágicas y trombóticas supuso un gasto de 11.445,17 euros. 
En ocasiones hay una inercia más que una resistencia en los profesionales que dificulta la actualización de las guías locales porque la rutina lleva a seguir aplicando recomendaciones que se utilizaron en el pasado.

Como en nuestro caso, se ha observado una disparidad entre las recomendaciones de las guías y los patrones de práctica clínica en Europa llegando a diseñarse programas electrónicos para ayudar en la aplicación correcta de las recomendaciones y mejorar la adhesión de los profesionales a las guías (125).

En nuestro Hospital y Centros de Atención Primaria seguíamos manejando guías de recomendaciones obsoletas y se estaba utilizando la terapia puente en pacientes en los que no era preciso esta profilaxis con heparina de bajo peso molecular.

Así, hemos constatado que la aplicación incorrecta de las guías y el uso de terapia puente con heparina de bajo peso molecular en pacientes de bajo riesgo provocó un aumento del sangrado tras el procedimiento invasivo.

De la misma manera, esta práctica supuso un incremento del coste, derivado del uso de heparina de bajo peso molecular y de la necesidad de acudir al Servicio de Urgencias o requerir ingreso hospitalario por presentar una complicación hemorrágica.

La incorrecta aplicación de las guías supone un riesgo para el paciente por lo que se debe hacer un esfuerzo para actualizar las guías locales y elaborar medidas de difusión de las nuevas recomendaciones.

De igual modo, debe efectuarse una valoración posterior del seguimiento que se ha hecho de las guías de tal manera que cualquier desviación de las mismas debe ser notificada a los profesionales para que sea corregida cuanto antes en beneficio de los pacientes y control del gasto. 
No solo la incorrecta aplicación de las guías o el incorrecto seguimiento de las recomendaciones supone un riesgo de yatrogenia para el paciente si no que el coste de la actuación se verá incrementado considerablemente.

Se debe hacer un esfuerzo por actualizar las guías locales de cada hospital y elaborar medidas de difusión de las nuevas recomendaciones tales como editar guías, presentar las actualizaciones en sesiones generales, en sesiones por servicios y así, dar a conocer los cambios que la investigación continua aporta en el campo de la anticoagulación.

Los hematólogos tenemos la responsabilidad de enseñar al resto de especialidades el adecuado manejo de los anticoagulantes tanto orales como subcutáneos y hacer seguir las recomendaciones.

Así mismo, debe efectuarse una valoración posterior del seguimiento que se ha hecho de las guías de tal manera que cualquier desviación de las mismas debe ser notificada a los profesionales para que sea corregida dicha desviación cuanto antes en beneficio de los pacientes. 
8. CONCLUSIONES 
1- No se siguieron las recomendaciones de la guía de la ACCP 2012, en las que se detalla que los pacientes con Fibrilación auricular de bajo riesgo no requieren sustitución del anticoagulante oral (acenocumarol) ante su retirada por un procedimiento invasivo programado.

2- Se utilizó terapia puente con heparina de bajo peso molecular a dosis profilácticas (enoxaparina $40 \mathrm{mg} /$ día vía subcutánea) en pacientes con Fibrilación auricular y bajo riesgo trombótico al suspender el anticoagulante oral, acenocumarol.

3- Se produjeron 5 complicaciones hemorrágicas: una mayor y cuatro menores al utilizar la terapia puente durante la retirada del anticoagulante oral (acenocumarol) ante un procedimiento invasivo programado.

4- Se produjo una complicación trombótica, un ictus en un paciente durante la retirada del anticoagulante oral (acenocumarol) ante un procedimiento invasivo programado.

5- La no implementación de las guías ACCP 2012 supuso un incremento del gasto farmacéutico por el uso de enoxaparina a dosis profilácticas durante la retirada del anticoagulante oral (acenocumarol) ante un procedimiento invasivo programado.

6- Así mismo, la no implementación de las guías ACCP 2012 supuso un incremento del gasto por el uso del Servicio de Urgencias y los días de ingreso que provocaron las complicaciones hemorrágicas secundarias al uso de enoxaparina profiláctica. 



\section{BIBLIOGRAFÍA}




\section{BIBLIOGRAFÍA}

1- Douketis JD, Spyropoulos AC, Spencer FA, et al. Perioperative management of antithrombotic therapy: antithrombotic therapy and prevention of thrombosis, 9th ed: American College of Chest Physicians Evidence-Based Clinical Practice Guidelines. Chest 2012; 141: 2 Suppl:e326Se350S.

2- Schofield FW. Damaged sweet clover; the cause of a new disease in cattle simulating hemorrhagic septicemia and blackleg. J AM Vet Med Ass. 1924; 64: 553.

3- Roderick LM. A problema in the coagulation of the blood: sweet clover disease of the cattle". Am J Physiol 1931; 96: 413.

4- Dam H. The antihemorrhagic vitamian of the chick. Biochem J. 1935; 29: 1273.

5- Binkley SB, Chenney LC, Holcomb WF. The constitution and synthesis of vitamin K. J Am Chem Soc. 1939; 61: 2558.

6- Quick AJ, Stanley-Brown M, Brancroft FW. A study of the coagulation defect in hemophilia and jaundice. Am J Med Sci. 1935; 190: 501.

7- Link KP. The discovery of dicumarol and its sequels. Circulation. 1959; 10: 97.

8- Whitlon DS, Sadowski JA, Suttie JW. Mechanism of coumarin action: significance of vitamin K epoxide reductase inhibition. Biochemistry. 1978; 17: 1371.

9- Butt HR, Allen EV, Billman JL. A preparation of spoiled sweet clover 3,3' methylenebis (4 $\mathrm{OH}$ coumarin) which prolongs coagulation and prothrombin time of the blood: preliminary report of experimental and clinical studies. Proceedings of the staff meetings of the Mayo Clinic. 1941; 16: 388.

10-Campbell HA, Roberts WL, Smith WK, Link KP. Studies of the hemorrhagic sweet clover disease: I. The preparation of hemorrhagic concentrates. J Biol Chem. 1940; 136: 47-55.

11-Huebner CF, Link KP. The syntesis of the delta-diketone derived from the hemorrhagic agent through alkaline degradation. J Biol Chem. 1941; 138: 529-534.

12-Butt HR, Allen EV, Billman JL. A preparation of spoiled sweet clover 3,3' methylenebis (4 $\mathrm{OH}$ coumarin) which prolongs coagulation and prothrombin time 
of the blood: preliminary report of experimental and clinical studies. Proceedings of the staff meetings of the Mayo Clinic. 1941; 16: 388-395.

13- Link KP. The discovery of dicumarol and its sequels. Circulation. 1959; 19: 97-107.

14-Shapiro S. Warfarin sodium derivative (coumadin sodium): intravenous hypoprothrombinemia-inducing agent. Angiology. 1953; 4: 380-390.

15-Clatanoff DV, Triggs PO, Meyer OO. Clinical experience with coumarin anticoagulants warfarin and warfarin sodium. Arch Int Med. 1954; 94: 213-220.

16-Pollock BE. Clinical experience with warfarin (coumadin)sodium, a new anticoagulant. JAMA. 1955; 159: 1094-1097.

17-Coon WW, Willis PW 3rd. Some aspects of the pharmacology of oral anticoagulants. ) Clin Pharmacol Ther. 1970; 11(3): 312-336.

18- Martínez Brotons F. Terapéutica antitrombótica. Sans-Sabrafen J. 5a edición. 2006.

19-Breckenridge AM, Leck JB, Park BK, Serlin MJ, Wilson A.Mechanisms of action of the anticoagulants warfarin, 2-chloro-3-phytylnaphthoquinone (Cl-K), acenocoumarol, brodifacoum and difenacoum in the rabbit [proceedings]. $\mathrm{Br} \mathrm{J}$ Pharmacol. 1978; 64(3): 399P.

20-Shearer MJ, Barkhan P. Vitamin K1 and therapy of massive warfarin overdose. Lancet. 1979; 1(8110): 266-267.

21-Schulman S1, Bijsterveld NR. Anticoagulants and their reversal. Transfus Med Rev. 2007; 21(1): 37-48.

22-Long LA, Colprong G, Marion P. A new 4-oxycoumarin derivative, G-23350 (sintrom). Can Med Assoc J. 1956; 75(4): 261-268.

23-Connell WF, Mayer GA. The anticoagulant effect of a new coumarin derivativesintrom (geigy)- and its control by standardized clotting time. Can Med Assoc J. 1957; 76(4): 272-278.

24- Jonhnson R, DavidD A, Chartier Y. Clinical experience with G-23350 (sintrom). Can Med Assoc J. 1957; 77(8): 756-761.

25-Quick AJ, Stanley-Brown m, Brancroft FW. A study of the coagulation defect in hemophilia and jaundice. AM J Med Sci. 1935; 190: 501-511.

26-Allen EV, Barker NW, Waugh JM. A preparation from spoiled sweet clover, 3,3' methylenebis ( $4 \mathrm{OH}$ coumarin) which prolongs coagulation and prothrombin time of the blood: a clinical study. JAMA. 1942; 120: 1009. 
27-Zucker S, Cathey MH, Sox PJ, Hall EC. Standardization of laboratory tests for controling anticoagulant therapy. Am J Clin Pathol. 1970; 53: 384.

28-Biggs R, Denson KW. Third report of the standardization of one-stage prothrombin time for the control of anticoagulant therapy. Thromb Diath Haemorrh. 1967; 26: 445.

29-Kirwood TB. Calibration of reference thromboplastins and standardization of the prothrombin time ratio. Thromb Haemostas. 1983; 49: 238.

30-ASHP Therapeutic Position Statement on the Use of the International Normalized Ratio System to Monitor Oral Anticoagulant Therapy. Am J Heath Syst Pharm. 1995; 52: 529-531.

31-Actualización y experiencias en la anticoagulación a largo plazo (TAO) en los sistemas de salud. Escuela Nacional de Sanidad, Instituto de Salud Carlos III 2003. 1985; 74: 125-131.

32-Vacas M, Fernández MA, Martínez-Brotons F, Lafuente PJ, Ripoll F, Alvarez C, Iriarte JA. Comparative study of a portable prothrombin time monitor employing three different systems in oral anticoagulant units. Haemostasis. 2001; 31(1): 18-25.

33-Ruzicka K, Kapiotis S, Quehenberger P et al. Evaluation of bedside prothrombin time and activated partial thromboplastin time measurement by coagulation analyzer CoaguCheck Plus in various clinical settings. Thromb Res. 1997; 87(5): 431440.

34-Cheung DS1, Heizer D, Wilson J, Gage BF.Cost-savings analysis of using a portable coagulometer for monitoring homebound elderly patients taking warfarin. Am J Geriatr Cardiol. 2003; 12(5): 283-287.

35-Lee JH1, Lee KS, Kim DS, Lee HS, Choi SI, Cho YG. Evaluation of CoaguChek XS for measuring prothrombin time in patients receiving long-term oral anticoagulant therapy. Korean J Lab Med. 2007; 27(3): 177-181 [Abstract].

36-Caballero-Villarraso J, Villegas-Portero R, Rodríguez-Cantalejo F. Portable coagulometer devices in the monitoring and control of oral anticoagulation therapy: a systematic review]. Aten Primaria. 2011; 43(3): 148-156.

37- Claes N1, Moeremans K, Frank B, Jef A, Jos V, Herman VL, Lieven A. Estimating the cost-effectiveness of quality-improving interventions in oral anticoagulation management within general practice. Value Health. 2006; 9(6): 369-376. 
38-Sharma P, Scotland G, Cruickshank M, Tassie E, Fraser C, Burton C, Croal B, Ramsay $\mathrm{CR}$, Brazzelli M. The clinical effectiveness and cost-effectiveness of point-of-care tests (CoaguChek system, INRatio2 PT/INR monitor and ProTime Microcoagulation system) for the self-monitoring of the coagulation status of people receiving longterm vitamin $\mathrm{K}$ antagonist therapy, compared with standard UK practice: systematic review and economic evaluation. Health Technol Assess. 2015; 19(48): 1-172. doi: 10.3310/hta19480.

39-Woods K, Douketis JD, Schnurr T, Kinnon K, Powers P, Crowther MA. Patient preferences for capillary vs. venous INR determination in an anticoagulation clinic: a randomized controlled trial. Thromb Res. 2004; 114(3): 161-165.

40-Wieloch M, Hillarp A, Strandberg K, Nilsson C, Svensson PJ. Comparison and evaluation of a Point-of-care device (CoaguChek XS) to Owren-type prothrombin time assay for monitoring of oral anticoagulant therapy with warfarin. Thromb Res. 2009; 124(3): 344-8. doi: 10.1016/j.thromres.2009.03.007.

41-Heneghan C, Alonso-Coello P, Garcia-Alamino JM, Perera R, Meats E, Glasziou P. Self-monitoring of oral anticoagulation: a systematic review and meta-analysis. Lancet. 2006; 367(9508): 404-411.

42-Testa S, Alatri A, Paoletti O, Morstabilini G, Medagliani MA, Denti N, Martellenghi E. Reorganisation of an anticoagulation clinic using a telemedicine system: description of the model and preliminary results. Intern Emerg Med. 2006; 1(1): 24-29.

43-Crighton G. Methods of coagulation. Methods Mol Biol. 2013; 992: 73-83. doi: 10.1007/978-1-62703-339-8_5.

44-Rosendaal FR, Cannegieter SC, Van der Meer FJ, Briët E. A method to determine the optimal intensity of oral anticoagulant therapy. Thromb Haemost. 1993; 69: 236-239.

45- Pisters R, Lane DA, Nieuwlaat R, De Vos CB, Crijns HJ, Lip GY. A novel user-friendly score (HAS-BLED) to assess 1-year risk of major bleeding in patients with atrial fibrillation: the Euro Heart Survey. Chest. 2010; 138: 1093-1100.

46-Kannel WB, Wolf PA, Benjamin EJ, Levy D. Prevalence, incidence, prognosis, and predisposing conditions for atrial fibrillation: population-based estimates. Am J Cardiol. 1998; 82(8A): 2N-9N. 
47-Thrall G, Lane D, Carroll D, Lip GY. Quality of life in patients with atrial fibrillation: a systematic review. (Am J Med. 2006; 119(5): 448.e1-19.

48-Benjamin EJ, Wolf PA, D'Agostino RB, Silbershatz H, Kannel WB, Levy D. Impact of atrial fibrillation on the risk of death: the Framingham Heart Study.Circulation. 1998; 98(10): 946-952.

49-Rahman F, Kwan GF, Benjamin EJ. Global epidemiology of atrial fibrillation. Nat Rev Cardiol. 2014; 11: 639-654.

50-Stewart S, Hart CL, Hole DJ, McMurray JJ. Population prevalence, incidence, and predictors of atrial fibrillation in the Renfrew/Paisley study. Heart. 2001; 86(5): 516-521.

51-Pérez-Villacastín J, Pérez Castellano N, Moreno Planas J. Epidemiology of atrial fibrillation in Spain in the past 20 years. Rev Esp Cardiol 2013; 66 (7): 561-565.

52-Redón J, Cea-Calvo L, Lozano JV, Martí-Canales JC, Llisterri JL, Aznar J, GonzálezEsteban J; Investigators of the PREV-ICTUS study. Blood pressure and estimated risk of stroke in the elderly population of Spain: the PREV-ICTUS study. Stroke. 2007; 38(4): 1167-1173.

53- Heeringa J, van der Kuip DA, Hofman A, Kors JA, van Herpen G, Stricker BH, Stijnen T, Lip GY, Witteman JC Prevalence, incidence and lifetime risk of atrial fibrillation: the Rotterdam study. Eur Heart J. 2006; 27(8): 949-953.

54-Barrios V, Calderon A, Escobar C, De la Figuera M, Grupo de Atención Primaria de la sección de Cardiología Clínica de la Sociedad Española de Cardiología. Pacientes con fibrilación auricular asistidos en consultas de atención primaria. Estudio ValFAAP. Rev Esp Cardiol. 2012; 65: 47-53.

55-Gómez-Doblas JJ, Muñiz J, Alonso Martin JJ, Rodríguez-Roca G, Lobos JM, Awamleh $P$, et al. Prevalencia de fibrilación auricular en España. Resultados del estudio OFRECE. Rev Esp Cardiol. 2014; 67: 259-269.

56- Morillas P, Pallarés V, Llisterri JL, Sanchis C, Sánchez T, Fácila L, et al. Prevalencia de fibrilación auricular y uso de fármacos antitrombóticos en el paciente hipertenso $\geq 65$ años. El registro FAPRES. Rev Esp Cardiol. 2010; 63: 943-950.

57-Kannel WB, Abbott RD, Savage DD, McNamara PM. Epidemiologic features of chronic atrial fibrillation: the Framingham study. N Engl J Med. 1982; 306(17): 1018-1022. 
58-Wolf PA, Dawber TR, Thomas HE Jr, Kannel WB. Epidemiologic assessment of chronic atrial fibrillation and risk of stroke: the Framingham study. Neurology. 1978; 28(10): 973-977.

59-Stroke Risk in Atrial Fibrillation Working Group. Independent predictors of stroke in patients with atrial fibrillation: a systematic review. Neurology. 2007; 69(6): 546554 [Abstract].

60-Masjuán J, Álvarez-Sabín J, Blanco M, De Felipe A, Gil-Núñez A, Gállego-Culleré J, et al. Manejo actual del tratamiento antitrombótico en pacientes con fibrilación auricular no valvular y antecedentes de ictus o ataque isquémico transitorio. Rev Neurol. 2014; 59: 25-36.

61-Benjamin EJ, Wolf PA, D'Agostino RB, Silbershatz H, Kannel WB, Levy D. Impact of atrial fibrillation on the risk of death: The framingham heart study. Circulation. 1998; 98: 946-952.

62-Wolf PA, Abbott RD, Kannel WB. Atrial fibrillation as an independent risk factor for stroke: the Framingham Study. Stroke. 1991; 22: 983-988.

63-Hart RG, Pearce LA, Aguilar MI, Meta-analysis:. antithrombotic therapy to prevent stroke in patients who have nonvalvular atrial fibrillation. Ann Intern Med. 2007; 146: 857-867.

64- Petersen P, Boysen G, Godtfredsen J, Andersen ED, Andersen B. Placebocontrolled, randomised trial of warfarin and aspirin for prevention of thromboembolic complications in chronic atrial fibrillation. The Copenhagen AFASAK study. Lancet. 1989; 1(8631): 175-179.

65-Stroke Prevention in Atrial Fibrillation Study Group Investigators. Preliminary report of the Stroke Prevention in Atrial Fibrillation Study. N Engl J Med. 1990; 322(12): 863-868.

66-Brandes A, Overgaard M, Plauborg L, Dehlendorff C, Lyck F, Peulicke J, Poulsen SV, Husted S. Guideline adherence of antithrombotic treatment initiated by general practitioners in patients with nonvalvular atrial fibrillation: a Danish survey. Clin Cardiol. 2013; 36(7): 427-432.

67-Atrial Fibrillation Investigators. Risk factors for stroke and efficacy of antithrombotic therapy in atrial fibrillation. Arch Intern Med. 1994; 154(13): 14491457. 
68-Gage BF, Waterman AD, Shannon W, Boechler M, Rich MW, Radford MJ. Validation of clinical classification schemes for predicting stroke: results from the National Registry of Atrial Fibrillation. JAMA. 2001; 285(22): 2864-2870.

69-Fuster V, Rydén LE, Cannom DS, Crijns HJ, Curtis AB, Ellenbogen KA, Halperin JL, Kay GN, Le Huezey JY, Lowe JE, Olsson SB, Prystowsky EN, Tamargo JL, Wann LS. 2011 ACCF/AHA/HRS focused updates incorporated into the ACC/AHA/ESC 2006 Guidelines for the management of patients with atrial fibrillation: a report of the American College of Cardiology Foundation/American Heart Association Task Force on Practice Guidelines developed in partnership with the European Society of Cardiology and in collaboration with the European Heart Rhythm Association and the Heart Rhythm Society. J Am Coll Cardiol. 2011 ; 57(11): e101-198.

70- Nieuwlaat R, Capucci A, Lip GY, Olsson SB, Prins MH, Nieman FH, López-Sendón J, Vardas PE, Aliot E, Santini M, Crijns HJ; Euro Heart Survey Investigators. Antithrombotic treatment in real-life atrial fibrillation patients: a report from the Euro Heart Survey on Atrial Fibrillation. Eur Heart J. 2006; 27(24): 3018-3026.

71-Lip GY, Nieuwlaat R, Pisters R, Lane DA, Crijns HJ. Refining clinical risk stratification for predicting stroke and thromboembolism in atrial fibrillation using a novel risk factor-based approach: the euro heart survey on atrial fibrillation. Chest. 2010 ; 137(2): 263-272.

72-Olesen JB, Torp-Pedersen C, Hansen ML, Lip GY. The value of the CHA2DS 2-VASC score for refining stroke risk stratification in patients with atrial fibrillation with a CHADS2 score 0-1: a nationwide cohort study. Thromb Haemost. 2012; 107(6): 1172-1179.

73- Mason PK, Lake DE, DiMarco JP, Ferguson JD, Mangrum JM, Bilchick K, Moorman LP, Moorman JR Impact of the CHA2DS 2-VASc score on anticoagulation recommendations for atrial fibrillation. Am J Med. 2012; 125(6): 603.e1-6.

74- Coppens M, Eikelboom JW, Hart RG, Yusuf S, Lip GY, Dorian P, Shestakovska O, Connolly SJ. The CHA2DS2-VASc score identifies those patients with atrial fibrillation and a CHADS2 score of 1 who are unlikely to benefit from oral anticoagulant therapy. Eur Heart J. 2013; 34(3): 170-176.

75- Fox KAA, Lucas JE, Pieper KS, Bassand JP, Camm AJ, Fitzmaurice DA, Goldhaber SZ, Goto S, Haas S, Hacke W, Kayani G, Oto A, Mantovani LG, Misselwitz F, Piccini JP, 
Turpie AGG, Verheugt FWA, Kakkar AK; GARFIELD-AF Investigators. Improved risk stratification of patients with atrial fibrillation: an integrated GARFIELD-AF tool for the prediction of mortality, stroke and bleed in patients with and without anticoagulation. BMJ Open. 2017; 7(12): e017157.

76- Flaherty ML, Kissela B, Woo D, Kleindorfer D, Alwell K, Sekar P, Moomaw CJ, Haverbusch M, Broderick JP. The increasing incidence of anticoagulant-associated intracerebral hemorrhage. Neurology. 2007; 68(2): 116-121.

77-Pisters R, Lane DA, Nieuwlaat R, de Vos CB, Crijns HJ, Lip GY. A novel user-friendly score (HAS-BLED) to assess 1-year risk of major bleeding in patients with atrial fibrillation: the Euro Heart Survey. Chest. 2010; 138(5): 1093-1100.

78-Apostolakis S, Lane DA, Guo Y, Buller H, Lip GYH. Performance of the HEMORR 2 HAGES, ATRIA, and HAS-BLED bleeding risk-prediction scores in nonwarfarin anticoagulated atrial fibrillation patients. J Am Coll Cardiol. 2013; 61(3): 386-387.

79-Omran H, Bauersachs R, Rübenacker S, Goss F, Hammerstingl C. The HAS-BLED score predicts bleedings during bridging of chronic oral anticoagulation. Results from the national multicentre BNK Online bRiDging REgistRy (BORDER). Thromb Haemost. 2012; 108(1): 65-73.

80- Fang MC, Go AS, Chang Y, Borowsky L, Pomernacki NK, Singer DE; ATRIA Study Group. Comparison of risk stratification schemes to predict thromboembolism in people with nonvalvular atrial fibrillation. J Am Coll Cardiol. 2008; 51(8): 810-815.

81-Roldán V, Marín F, Fernández H, Manzano-Fernandez S, Gallego P, Valdés M, Vicente V, Lip GYH. Predictive value of the HAS-BLED and ATRIA bleeding scores for the risk of serious bleeding in a "real-world" population with atrial fibrillation receiving anticoagulant therapy. Chest. 2013; 143(1): 179-184.

82-Camm AJ, Lip GY, De Caterina R, Savelieva I, Atar D, Hohnloser SH, Hindricks G, Kirchhof P; ESC Committee for Practice Guidelines-CPG; Document Reviewers. 2012 focused update of the ESC Guidelines for the management of atrial fibrillation: an update of the 2010 ESC Guidelines for the management of atrial fibrillation--developed with the special contribution of the European Heart Rhythm Association. Europace. 2012; 14(10): 1385-1413.

83-January CT, Wann LS, Calkins H, Chen LY, Cigarroa JE, Cleveland JC Jr, Ellinor PT, Ezekowitz MD, Field ME, Furie KL, Heidenreich PA, Murray KT, Shea JB, Tracy CM, 
Yancy CW. 2019 AHA/ACC/HRS Focused Update of the 2014 AHA/ACC/HRS

Guideline for the Management of Patients With Atrial Fibrillation: A Report of the American College of Cardiology/American Heart Association Task Force on Clinical Practice Guidelines and the Heart Rhythm Society. J Am Coll Cardiol. 2019; 74(1): 104-132.

84-Senoo K, Lau YC, Lip GY. Updated NICE guideline: management of atrial fibrillation (2014). Expert Rev Cardiovasc Ther. 2014; 12(9): 1037-1040.

85-Wysokinski WE, McBane RD. Periprocedural bridging management of anticoagulation. Circulation. 2012; 126(4): 486-490.

86-Guyatt GH, Akl EA, Crowther M, Gutterman DD, Schuünemann HJ; American College of Chest Physicians Antithrombotic Therapy and Prevention of Thrombosis Panel. Executive summary: Antithrombotic Therapy and Prevention of Thrombosis, 9th ed: American College of Chest Physicians Evidence-Based Clinical Practice Guidelines. Chest. 2012 Feb;141(2 Suppl):7S-47S.

87-Siegal D, Yudin J, Kaatz S, Douketis JD, Lim W, Spyropoulos AC. Periprocedural heparin bridging in patients receiving vitamin $\mathrm{K}$ antagonists: systematic review and meta-analysis of bleeding and thromboembolic rates. Circulation. 2012; 126(13): 1630-1639.

88-Steinberg BA, Peterson ED, Kim S, Thomas L, Gersh BJ, Fonarow GC, Kowey PR, Mahaffey KW, Sherwood MW, Chang P, Piccini JP, Ansell J; Outcomes Registry for Better Informed Treatment of Atrial Fibrillation Investigators and Patients. Use and outcomes associated with bridging during anticoagulation interruptions in patients with atrial fibrillation: findings from the Outcomes Registry for Better Informed Treatment of Atrial Fibrillation (ORBIT-AF).Circulation. 2015; 131(5): 488-494.

89-Douketis JD, Spyropoulos AC, Kaatz S, Becker RC, Caprini JA, Dunn AS, Garcia DA, Jacobson A, Jaffer AK, Kong DF, Schulman S, Turpie AG, Hasselblad V, Ortel TL; BRIDGE Investigators. Perioperative Bridging Anticoagulation in Patients with Atrial Fibrillation. N Engl J Med. 2015; 373(9): 823-833.

90-Laurent TC, Tengblad A, Thunberg L, Hök M, Lindahl U. The molecular-weightdependence of the anti-coagulant activity of heparin. Biochem J. 1978; 175(2): 691-701. 
91-Kakkar VV, Djazaeri B, Fok J, Fletcher M, Scully MF, Westwick J. Low-molecularweight heparin and prevention of postoperative deep vein thrombosis. $\mathrm{Br}$ Med J (Clin Res Ed). 1982; 284(6313): 375-379.

92-Turpie AG, Levine MN, Hirsh J, Carter CJ, Jay RM, Powers PJ, Andrew M, Hull RD, Gent M. A randomized controlled trial of a low-molecular-weight heparin (enoxaparin) to prevent deep-vein thrombosis in patients undergoing elective hip surgery. N Engl J Med. 1986; 315(15): 925-929.

93- Johnson EA, Kirkwood TBL, Stirling Y, Perez-Requejo J L Four heparin preparations: Anti-Xa potentiating effect of heparin after subcutaneous injection. Thromb Haemost. 1976; 35: 586.

94-Andersson L-0, Barrowcliffe TW, Holmer E, Johnson EA, Sims GEC: Anticoagulant properties of heparin fractionated by affinity chromatography on matrix-bound antithrombin 111 and by gel filtration. Thromb Res. 1976; 9: 575.

95-Carter CJ, Kelton JG, Hirsh J, Cerskus AL, Santos AV, Gent M: The relationship between the hemorrhagic and antithrombotic properties of low molecular weight heparins and heparin. Blood. 1982; 59: 1239.

96- Hirsh J, Levine MN. Low molecular weight heparin. Blood. 1992; 79(1): 1-17.

97-Pengo V, Cucchini U, Denas G, Erba N, Guazzaloca G, La Rosa L et al. Standardized Low-Molecular-Weight Heparin Bridging Regimen in Outpatients on Oral Anticoagulants Undergoing Invasive Procedure or Surgery An Inception Cohort Management Study. Circulation. 2009; 119: 2920-2927.

98-Sureda A, García Frade LJ, Navarro JL. Nuevas opciones en la prevención y tratamiento de la enfermedad tromboembólica: las heparinas de bajo peso molecular. Sangre. 1990; 35: 389-396.

99-Tapson VF. Deep venous thrombosis: outpatient therapy with low-molecularweight heparin. Manag Care. 1999; 8 Suppl: 2-6.

100- Chong BH, Brighton TA, Baker RI, Thurlow P, Lee CH; ASTH DVT Study Group. Once-daily enoxaparin in the outpatient setting versus unfractionated heparin in hospital for the treatment of symptomatic deep-vein thrombosis. J Thromb Thrombolysis. 2005; 19(3): 173-181.

101- Marlovits S, Striessnig G, Schuster R, Stocker R, Luxl M, Trattnig S, Vécsei V. Extended-duration thromboprophylaxis with enoxaparin after arthroscopic surgery 
of the anterior cruciate ligament: a prospective, randomized, placebo-controlled study. Arthroscopy. 2007; 23(7): 696-702.

102- Wade WE, Hawkins DW. Cost effectiveness of outpatient anticoagulant prophylaxis after total hip arthroplasty. Orthopedics. 2000 ; 23(4): 335-338.

103- Haas SK. Venous thromboembolic risk and its prevention in hospitalized medical patients. Semin Thromb Hemost. 2002; 28(6): 577-584.

104- Kröger K. Prophylaxis of deep vein thrombosis with enoxaparin $40 \mathrm{mg}$ in outpatients compared to hospitalized medically ill patients. Med Klin (Munich). $2009 ; 104(8):$ 608-613.

105- Alberca F, Marín F, Roldán V, Carballo F. Manejo de los fármacos antitrombóticos asociados a los procedimientos digestivos. Rev Esp Enferm Dig. 2015; 107: 289-306.

106- Kristensen SD, Knuuti J, Saraste A, et al. 2014 ESC/ESA Guidelines on noncardiac surgery: cardiovascular assessment and management. Eur Heart J. 2014; 35: 2383-2431.

107- Rechenmacher SJ, Fang JC. Bridging anticoagulation: primum non nocere. J Am Coll Cardiol. 2015; 66: 1392-1403.

108- Spyropoulos AC, Al-Badri A, Sherwood MW, Douketis JD. Periprocedural management of patients receiving a vitamin $\mathrm{K}$ antagonist or a direct oral anticoagulant requiring an elective procedure or surgery. J Thromb Haemost. 2016; 14: 875-885.

109- Birnie DH, Healey JS, Wells GA, et al. BRUISE CONTROL Investigators. Pacemaker or defibrillator surgery without interruption of anticoagulation. N Engl J Med. 2013; 368: 2084-2093.

110- Doherty JU, Gluckman TJ, Hucker WJ, et al. 2017 ACC expert consensus decisión pathway for periprocedural management of anticoagulation in patients with nonvalvular atrial fibrillation: a report of the American College of Cardiology Clinical Expert Consensus Document Task Force. J Am Coll Cardiol. 2017; 69: 871898.)

111- Kirchhof P, Benussi S, Kotecha D, et al.2016 ESC Guidelines for the management of atrial fibrillation developed in collaboration with EACTS. Eur Heart J. 2016; 37: 2893-2962. 
112- Perry JD, Noakes TJ, Helliwell PS; British Dental Society. Guidelines for the management of patients on oral anticoagulants requiring dental surgery. Br Dent J. 2007; 203: 389-393.

113- Steinberg BA, Peterson ED, Kim S, et al. Use and outcomes associated with bridging during anticoagulation interruptions in patients with atrial fibrillation: findings from the Outcomes Registry for Better Informed Treatment of Atrial Fibrillation(ORBIT-AF). Circulation. 2015; 131: 488-494.

114- Clark NP, Witt DM, Davies LE, Saito EM, McCool KH, Douketis JD, Metz KR, Delate T. Bleeding, Recurrent Venous Thromboembolism, and Mortality Risks During Warfarin Interruption for Invasive Procedures. JAMA Intern Med. 2015; 175(7): 1163-1168.

115- van Veen JJ, Makris M. Management of peri-operative anti-thrombotic therapy. Anaesthesia. 2015; 70 (Suppl 1): 58-67.

116- Hillis CM, Schimmer AD, Couban S, Crowther MA. The Canadian Choosing Wisely campaign: the Canadian Hematology Society's top five tests and treatments. Ann Hematol. 2015; 94(4): 541-545.

117- Ríos SJ, Rivera-Caravaca JM, Aroca-Valverde C, Reguilón-Gallego L, CastilloRodríguez M, Esteve-Pastor MA, Vicente V, Marín F, Roldán V. Efficacy and safety of peri-procedural bridging therapy with low molecular weight heparin in atrial fibrillation patients under vitamin K antagonists. QJM. 2019; 112(3): 183-188.

118- van der Pol S, Jacobs MS, Meijer K, Piersma-Wichers MG, Tieleman RG, Postma MJ, van Hulst $M$. Perioperative bridging of vitamin $K$ antagonist treatment in patients with atrial fibrillation: only a very small group of patients benefits. Europace. 2019; 21(5): 716-723.

119- Barrios V, Escobar C, Prieto L, Lobos JM, Polo J, Vargas D. Control of Anticoagulation With Warfarin or Acenocoumarol in Spain. Do They Differ?. Rev Esp Cardiol (Engl Ed). 2015; 68(12): 1181-1182.

120- Alonso Roca R, Figueroa Guerrero CA, Mainar de Paz V, Arribas García MP, Sánchez Perruca L, Rodríguez Barrientos R, Casado López M, Pedraza Flechas AM. Quality control of oral anticoagulant therapy in Primary Care in Madrid City, Spain: CHRONOS-TAO study. Med Clin (Barc). 2015; 145(5): 192-197. 
121- Liu S, Singer A, McAlister FA, Peeler W, Heran BS, Drummond N, Manca DP, Allan GM, Korownyk C, Kolber MR, Greiver M, Garrison SR. Quality of warfarin management in primary care: Determining the stability of international normalized ratios using a nationally representative prospective cohort. Can Fam Physician. 2019; 65(6): 416-425.

122- Parry D, Bryan S, Gee K, Murray E, Fitzmaurice D. Patient costs in anticoagulation management: a comparison of primary and secondary care. $\mathrm{Br} \mathrm{J}$ Gen Pract. 2001; 51(473): 972-976.

123- Pappas MA, Barnes GD, Vijan S. Cost-Effectiveness of Bridging Anticoagulation Among Patients with Nonvalvular Atrial Fibrillation. J Gen Intern Med. 2019; 34(4): 583-590.

124- Pelkofski EB, Baker WD, Rowlingson JC, Cantrell LA, Duska LR. Quality Initiative to Improve Compliance With Perioperative Anticoagulation. J Oncol Pract. 2019; 15(9): e835-e842.

125- Deharo JC, Sciaraffia E, Leclercq C et al. Coordinated by the Scientific Initiatives Committee of the European Heart Rhythm Association Perioperative management of antithrombotic treatment during implantation or revision of cardiac implantable electronic devices: the European Snapshot Survey on Procedural Routines for Electronic Device Implantation (ESS-PREDI). Europace. 2016; 18(5): 778-784. 